\title{
Colour distributions in E-S0 galaxies
}

\section{Colour data and dust in E's from Nieto's $B, R$ frames ${ }^{\star}$}

\author{
R. Michard \\ 1 Observatoire de la Côte d'Azur, Dept. Augustin Fresnel, BP. 229, F-06304 Nice Cedex 4, France \\ 2 Observatoire de Paris, DEMIRM, 77 Av. Denfert-Rochereau, F-75015 Paris, France
}

Received October 23, 1998; accepted March 9, 1999

\begin{abstract}
The $B-R$ colours distributions (with $R$ in Cousins's system) have been measured in 44 E classified galaxies in the Local Supercluster, from pairs of frames collected by Nieto and co-workers in 1989-91. These are nearly all from the CFHT, and of sub-arsec resolution.

Great attention has been given to the effects of unequal PSF's in the $B$ and $R$ frames upon colour distributions near centre; such effects are illustrated from model calculations and from pseudo-colours obtained from pairs of frames taken in the same band but with different seeing conditions. Appropriate corrections were systematically applied in order to derive central colours and inner gradients, although still affected by the limited resolution of the frames.
\end{abstract}

The radial colour distributions have been measured in more detail than usual, considering separately the near major axis and near minor axis regions of the isophotal contours. Azimuthal colour distributions, in rings limited by selected isophotes, were also obtained.

Dust "patterns", i.e. patches, lanes, arcs, ..., have been detected and mapped from the colour distributions. An ad hoc dust pattern importance index (or DPII) in a scale of 0 to 3 , has been introduced to qualify their size and contrast.

We have tried to find evidence of a diffuse dust concentration towards the disk, if one is apparent. Positive results (noted by the $d d$ symbol) have been obtained for disky E's, whenever the inclination of their disk to the line of sight is large enough, and eventually also in the small isolated disks sometimes present in both boxy and disky galaxies.

The red central peak occurring in many E-galaxies might be the signature of a central concentration of dust, also in cases where this peak is isolated rather than embedded in some extensive colour pattern. The properties

Send offprint requests to: R. Michard

* Based on observations collected at the Canada-FranceHawaii Telescope and at the Observatoire du Pic du Midi. of the near centre colour profiles have been related to a classification of nuclear photometric profiles into "flat topped" and "sharply peaked" (equivalent to "core-like" and "power-law" in the terminology of Faber et al. 1997).

The published here data include the following:

. Short descriptions and codes for the characters of the $B-R$ distribution of each object, and comparison to the results of recent surveys.

. A table of the mean $B-R$ at the centre and at two selected isophotes, a "core colour gradient" and the usual logarithmic gradient.

. Maps of near core $B-R$ isochromes and $B$ isophotes for comparison.

Images of the $B-R$ colour distribution are made available in electronic form.

Key words: galaxies: elliptical and lenticulars, CD galaxies: ISM - galaxies: fundamental parameters

\section{Introduction}

In 1989-91, the late J.-L. Nieto and co-workers obtained at the CFHT frames of many elliptical galaxies, plus a few S0 objects, in order to investigate the core profiles of these objects. Preliminary results were published by Nieto et al. (1991a, 1991b). They found that the cores of E-galaxies could be empirically sorted out into two classes, one well or nearly resolved at the CFHT resolution, the other remaining quite unresolved: the disky ellipticals (or diE), like the S0's, were in the sharp nucleus class. The problem of galaxian central profiles has now been tackled successfully by several groups using HST frames, with special emphasis upon E-S0 galaxies, providing more quantitative confirmations of the conjectures of Nieto's et al. However core colour profiles and patterns of such objects have not yet been much studied at high resolution. It was therefore 
deemed useful to analyse the best $B$ and $R$ frames in Nieto's CFHT collection, restricting the sample to relatively nearby galaxies, i.e. objects in the Local Supercluster with $V_{0}<3000 \mathrm{~km} \mathrm{~s}^{-1}$. Suitable pairs for our goal were available for 38 E-type galaxies, that is objects so classified in one of the usual catalogues. The data was supplemented by Pic du Midi $B, R$ frames for 6 E objects, obtained by E. Davoust and kindly put at our disposal.

In previous papers of this series some results were presented, based largely or exclusively upon the present "Nieto's sample". Such results are shortly summarized below. These papers also described the background and purposes of our work. The corresponding comments and references will not be repeated here. Review papers of interest have been published by Goudfrooij (1995) and (1996).

Our previous results are here recalled:

1. In Paper I of this series (Michard 1998a) a dust pattern importance index (or DPII) was obtained for a nearly complete sample of 67 ellipticals, using the present Nieto's sample supplemented by literature data. The frequency and importance of dust patterns for various brands of E-classified galaxies were studied from statistics of this index. It was found that dust is much more frequently detected, and with more important patterns, among diE's than boE's, the unE's beeing intermediate.

2. In Paper II (Michard 1998b) evidence for dust concentration in the disk was sought, for galaxies containing such a component: the criteria were an asymmetry in light and colour along the minor axis, as described for S0's by Michard \& Simien (1993) (MS93). and/or a flattening of the isochromes as compared to the isophotes. In case of positive evidence, the symbol $d d$ for "dust in disk" was introduced. This occurs in diE's, whenever the inclination of their disk to the line of sight is large enough, and eventally also in the small isolated disks sometimes present in both boE's and diE's galaxies.

3. In Paper III (Michard 1998c) the colour amplitude of the central red peak detected in most E-galaxies was described by the parameter $\Delta C_{0,3}$, i.e. the difference of colour between the centre and the isophote of mean radius 3 arcsec. For a sample of 39 galaxies, the central profiles were classified into two classes, shown to be equivalent to the "core-like" and "power-law" types of Faber et al. (1997). It was found that the red peak amplitude is very small for "core-like" objects, unless they show clear evidence of a dust pattern. This agrees with recent HST results by Carollo et al. (1997), from a special subsample of objects with kinematically decoupled cores, and possibly rule out a model for the formation of flat cores proposed by Silva \& Wise (1996).

This paper will present our data in more detail than in the previously published papers. Section 2 will describe the observations. Section 3 will introduce the techniques to be used in order to derive reliable $1 \mathrm{D}$ and $2 \mathrm{D}$ colour distributions. A special emphasis is given to errors induced by the different PSF's of the two frames involved in colour measurements, and to the techniques applied to reduce such errors. Section 4 describes various typical features of the colour distributions in E-galaxies, and introduces useful ad hoc parameters. Section 5 compiles the data of interest for statistical discussions and presents comparisons with other works.

Further papers of this series will deal with:

- An examination of colour distributions in strongly inclined S0 galaxies, a project started in cooperation with Dr. P. Poulain in Toulouse. The available material for this kind of objects is rather scanty, except for the study of NGC 3115 by Silva et al. (1989), and the observations of bulges by Balcells \& Peletier (1994).

Statistics of the colour material gathered at Observatoire de Haute-Provence in collaboration with the late J. Marchal.

\subsection{Notations and abbreviations}

It may be useful to collect here the notations and abbreviations currently used below.

. SuBr surface brightness.

. $\operatorname{maj} A, \min A$, major and minor axis respectively

. $a, c$ major and minor axis of the Reference Ellipse for the representation of an isophote according to Carter (1978).

$r=(a c)^{1 / 2}$ mean radius of the isophote.

. $e_{i}, f_{i}$ coefficients of cosine and sine terms in Carter's harmonic representation of deviations from the Reference Ellipse.

. PA position angle of major axis.

. $q$ axis ratio of the Reference Ellipse.

. $\epsilon=1-q$ ellipticity.

. diE, boE, unE subclassification of ellipticals as disky, boxy or undeterminate; $p$ added for peculiar envelopes.

\section{Observations}

Two set of observations are used in this survey. The first is from 4 runs at the CFHT in 1989-91, the second from one night at the TBL (Telescope Bernard Lyot) of the Pic du Midi. Tables 1 and 2 list the used frames with some relevant informations. Since the frames were intended to measure central light profiles, the exposure times were generally short, and many frames are of rather poor $S / N$ ratio. A classification of $S / N$ in terms of G (good), F (fair) and $\mathrm{P}$ (poor) is given in the table. 
Table 1. List of Cassegrain CFHT frames used in this work. $F=$ filter. Exposure times are in seconds. $W=F W H M$ in arcsec. Code for $S / N: \mathrm{G}=$ good; $\mathrm{F}=$ fair $\mathrm{P}=$ poor. $R=$ approximate range of measured radius in arcsec. When the file code contains an $m$, it was obtained by merging two frames. Individual notes: NGC 0821: nearby bright star. NGC 3115: Frames used here for technical tests

\begin{tabular}{|c|c|c|c|c|c|c|c|}
\hline NGC & $F$ & File & Date & Exp & $W$ & $\overline{S / N}$ & $R$ \\
\hline \multirow[t]{2}{*}{0584} & $B$ & h051 & $05 / 12 / 89$ & 180 & 1.15 & $\mathrm{G}$ & 30 \\
\hline & $R$ & h051 & id & 60 & 1.02 & G & 30 \\
\hline \multirow[t]{2}{*}{0596} & $B$ & h051 & $05 / 12 / 89$ & 210 & 1.10 & G & 30 \\
\hline & $R$ & h051 & id & 90 & 0.75 & $\mathrm{G}$ & 30 \\
\hline \multirow[t]{2}{*}{0636} & $B$ & h051 & $05 / 12 / 89$ & 300 & 1.13 & $\mathrm{G}$ & 30 \\
\hline & $R$ & h051 & id & 150 & 0.96 & G & 30 \\
\hline \multirow[t]{2}{*}{0720} & $B$ & h051 & $05 / 12 / 89$ & 180 & - & G & 20 \\
\hline & $R$ & h051 & id & 60 & - & $\mathrm{G}$ & 20 \\
\hline \multirow[t]{2}{*}{0821} & $B$ & h051 & $05 / 12 / 89$ & 300 & 0.90 & $\mathrm{G}$ & 20 \\
\hline & $R$ & h051 & id & 120 & 0.87 & $\mathrm{G}$ & 20 \\
\hline \multirow[t]{2}{*}{1052} & $B$ & h051 & $05 / 12 / 89$ & 180 & 0.96 & G & 25 \\
\hline & $R$ & h051 & id & 60 & 0.82 & $\mathrm{G}$ & 25 \\
\hline \multirow[t]{2}{*}{2768} & $B$ & h062 & $06 / 12 / 89$ & 300 & 1.00 & G & 20 \\
\hline & $R$ & h063 & id & 120 & 0.66 & G & 20 \\
\hline \multirow[t]{2}{*}{2974} & $B$ & h062 & $06 / 12 / 89$ & 120 & 0.68 & $F$ & 20 \\
\hline & $R$ & h06m & id & 90 & 0.68 & $\mathrm{G}$ & 20 \\
\hline \multirow[t]{2}{*}{3115} & $R$ & h051 & $05 / 12 / 89$ & 30 & 0.91 & $F$ & 40 \\
\hline & $R$ & h064 & $06 / 12 / 89$ & 60 & 0.55 & G & 35 \\
\hline \multirow[t]{5}{*}{3377} & $B$ & h052 & $05 / 12 / 89$ & 150 & - & G & 40 \\
\hline & $R$ & h052 & id & 150 & - & $\mathrm{G}$ & 40 \\
\hline & $B$ & h061 & $06 / 12 / 89$ & 45 & 0.76 & $F$ & 20 \\
\hline & $R$ & h062 & id & 15 & 0.59 & $F$ & 20 \\
\hline & $R$ & h063 & id & 15 & 0.66 & $F$ & 20 \\
\hline \multirow[t]{2}{*}{3379} & $B$ & h06m & $06 / 12 / 89$ & 60 & 0.81 & $F$ & 30 \\
\hline & $R$ & h06m & id & 30 & 0.60 & $F$ & 30 \\
\hline \multirow[t]{2}{*}{3585} & $B$ & h052 & $05 / 12 / 89$ & 180 & 1.16 & $\mathrm{G}$ & 30 \\
\hline & $R$ & h052 & id & 90 & 1.07 & $\mathrm{G}$ & 30 \\
\hline \multirow[t]{4}{*}{3610} & $B$ & h061 & $06 / 12 / 89$ & 90 & 0.72 & $F$ & 20 \\
\hline & $R$ & h061 & id & 15 & 0.52 & $\mathrm{P}$ & 20 \\
\hline & $R$ & h062 & id & 20 & 0.59 & $\mathrm{P}$ & 20 \\
\hline & $R$ & h063 & id & 60 & 0.60 & $F$ & 20 \\
\hline \multirow[t]{2}{*}{3613} & $B$ & h061 & $06 / 12 / 89$ & 30 & 0.80 & $\mathrm{P}$ & 10 \\
\hline & $R$ & h061 & id & 90 & 0.55 & $F$ & 10 \\
\hline \multirow[t]{2}{*}{4125} & $B$ & h042 & $04 / 04 / 90$ & 120 & 0.91 & $F$ & 20 \\
\hline & $R$ & h042 & id & 45 & 0.77 & $F$ & 20 \\
\hline \multirow[t]{2}{*}{4365} & $B$ & h042 & $04 / 04 / 90$ & 120 & 0.82 & $F$ & 25 \\
\hline & $R$ & h042 & id & 45 & 0.96 & $F$ & 25 \\
\hline \multirow[t]{2}{*}{4374} & $B$ & h041 & $04 / 04 / 90$ & 120 & - & $F$ & 30 \\
\hline & $R$ & h042 & id & 15 & - & $F$ & 30 \\
\hline \multirow[t]{3}{*}{4387} & $B$ & h042 & $04 / 04 / 90$ & 120 & 1.03 & $F$ & 20 \\
\hline & $R$ & h042 & id & 60 & 0.70 & G & 20 \\
\hline & $R$ & h043 & id & 120 & 0.69 & G & 20 \\
\hline \multirow[t]{2}{*}{4406} & $B$ & h041 & $04 / 04 / 90$ & 120 & 0.67 & $F$ & 20 \\
\hline & $R$ & h041 & id & 45 & 0.74 & $F$ & 20 \\
\hline 4472 & $B$ & h042 & $04 / 04 / 90$ & 120 & 0.77 & $F$ & 30 \\
\hline & $R$ & h042 & id & 45 & 0.72 & $F$ & 30 \\
\hline 4473 & $B$ & $\mathrm{~h} 04 \mathrm{~m}$ & $04 / 04 / 90$ & 135 & - & $F$ & 20 \\
\hline & $R$ & h042 & id & 45 & - & $F$ & 20 \\
\hline 4478 & $B$ & h043 & $04 / 04 / 90$ & 300 & 0.87 & $F$ & 20 \\
\hline & $R$ & h042 & id & 45 & 0.71 & $F$ & 20 \\
\hline 4494 & $B$ & $\mathrm{~h} 04 \mathrm{~m}$ & $04 / 04 / 90$ & 135 & 1.20 & $F$ & 20 \\
\hline & $R$ & h041 & id & 45 & 0.75 & $F$ & 20 \\
\hline
\end{tabular}

Table 2. List of HRCam CFHT frames used in this work. See previous table for explanations. Individual notes: NGC 4660 frame h073: a defect near the galaxian core could be corrected using another frame of lesser $S / N$

\begin{tabular}{|c|c|c|c|c|c|c|c|}
\hline NGC & $\bar{F}$ & File & Date & Exp & $W$ & $S / N$ & $R$ \\
\hline \multirow[t]{2}{*}{4261} & $B$ & h202 & $20 / 04 / 91$ & 300 & 0.77 & $\overline{\mathrm{P}}$ & 25 \\
\hline & $R$ & h202 & id & 60 & 0.80 & $\mathrm{P}$ & 25 \\
\hline \multirow[t]{3}{*}{4406} & $B$ & h202 & $20 / 04 / 91$ & 300 & 0.85 & $\mathrm{~F}$ & 35 \\
\hline & $R$ & h202 & id & 120 & 0.72 & $\mathrm{~F}$ & 35 \\
\hline & $R$ & h203 & id & 90 & 0.61 & $\mathrm{~F}$ & 35 \\
\hline \multirow[t]{4}{*}{4472} & $B$ & h202 & $20 / 04 / 91$ & 300 & 0.96 & $\mathrm{~F}$ & 25 \\
\hline & $B$ & h203 & id & 300 & 0.82 & $\mathrm{~F}$ & 25 \\
\hline & $R$ & h202 & id & 120 & 0.86 & $\mathrm{~F}$ & 25 \\
\hline & $R$ & h204 & id & 90 & 0.72 & $\mathrm{~F}$ & 25 \\
\hline \multirow[t]{2}{*}{4494} & $B$ & h202 & $20 / 04 / 91$ & 300 & 0.89 & $\mathrm{~F}$ & 30 \\
\hline & $R$ & h202 & id & 60 & 0.77 & $\mathrm{~F}$ & 30 \\
\hline \multirow[t]{2}{*}{4551} & $B$ & h212 & $21 / 04 / 91$ & 240 & - & $\mathrm{P}$ & 20 \\
\hline & $R$ & h212 & id & 90 & - & $\mathrm{P}$ & 20 \\
\hline \multirow[t]{2}{*}{4564} & $B$ & h212 & $21 / 04 / 91$ & 180 & - & $\mathrm{F}$ & 25 \\
\hline & $R$ & h212 & id & 90 & - & $\mathrm{F}$ & 25 \\
\hline \multirow[t]{2}{*}{4621} & $B$ & h203 & $21 / 04 / 91$ & 210 & 0.81 & $\mathrm{~F}$ & 15 \\
\hline & $R$ & h203 & id & 30 & 0.85 & $\mathrm{~F}$ & 15 \\
\hline \multirow[t]{2}{*}{4742} & $B$ & h192 & $19 / 04 / 91$ & 240 & 0.77 & $\mathrm{~F}$ & 15 \\
\hline & $R$ & h191 & id & 45 & 0.61 & $\mathrm{~F}$ & 15 \\
\hline \multirow[t]{2}{*}{5322} & $B$ & h202 & $20 / 04 / 91$ & 480 & 0.89 & $\mathrm{~F}$ & 15 \\
\hline & $R$ & h204 & id & 150 & 0.78 & $\mathrm{~F}$ & 15 \\
\hline \multirow[t]{2}{*}{5813} & $B$ & $\mathrm{~h} 20 \mathrm{~m}$ & $20 / 04 / 91$ & 450 & 0.84 & $\mathrm{~F}$ & 15 \\
\hline & $R$ & h203 & id & 150 & 0.75 & $\mathrm{~F}$ & 15 \\
\hline \multirow[t]{2}{*}{5845} & $B$ & h212 & $21 / 04 / 91$ & 120 & 0.66 & $\mathrm{P}$ & 10 \\
\hline & $R$ & h213 & id & 60 & 0.48 & $\mathrm{P}$ & 10 \\
\hline \multirow[t]{2}{*}{5846} & $B$ & h212 & $21 / 04 / 91$ & 120 & 0.70 & $\mathrm{P}$ & 15 \\
\hline & $R$ & h213 & id & 15 & 0.61 & $\mathrm{P}$ & 15 \\
\hline \multirow[t]{3}{*}{$5846 \mathrm{~A}$} & $B$ & $\mathrm{~h} 21 \mathrm{~m}$ & $21 / 04 / 91$ & 210 & 0.70 & $\mathrm{P}$ & 8 \\
\hline & $R$ & h214 & id & 120 & 0.61 & $\mathrm{~F}$ & 8 \\
\hline & $R$ & h216 & id & 45 & 0.64 & $\mathrm{P}$ & 8 \\
\hline \multirow[t]{2}{*}{4478} & $\bar{B}$ & h072 & $07 / 06 / 91$ & 180 & 0.62 & $\mathrm{P}$ & 20 \\
\hline & $R$ & 072 & id & 60 & 0.50 & $\mathrm{~F}$ & 20 \\
\hline \multirow[t]{2}{*}{$4486 \mathrm{~B}$} & $B$ & h072 & $07 / 06 / 91$ & 120 & 0.71 & $\mathrm{P}$ & 6 \\
\hline & $R$ & h072 & id & 30 & 0.57 & $\mathrm{P}$ & 6 \\
\hline \multirow[t]{2}{*}{4649} & $B$ & h072 & $07 / 06 / 91$ & 240 & 0.69 & $\mathrm{~F}$ & 10 \\
\hline & $R$ & h072 & id & 150 & 0.65 & $\mathrm{~F}$ & 10 \\
\hline \multirow[t]{3}{*}{4660} & $B$ & h073 & $07 / 06 / 91$ & 180 & 0.81 & $\mathrm{~F}$ & 15 \\
\hline & $B$ & $\mathrm{~h} 07 \mathrm{~m}$ & id & 180 & 0.81 & $\mathrm{~F}$ & 15 \\
\hline & $R$ & h073 & id & 60 & 0.73 & $\mathrm{~F}$ & 15 \\
\hline \multirow[t]{2}{*}{5576} & $B$ & h072 & $07 / 06 / 91$ & 180 & 0.99 & $\mathrm{~F}$ & 15 \\
\hline & $R$ & h072 & id & 60 & 0.60 & $\mathrm{~F}$ & 15 \\
\hline \multirow[t]{2}{*}{5638} & $B$ & h072 & $07 / 06 / 91$ & 180 & 0.90 & $\mathrm{P}$ & 15 \\
\hline & $R$ & h072 & id & 120 & 0.69 & $\mathrm{~F}$ & 15 \\
\hline \multirow[t]{2}{*}{5831} & $B$ & $\mathrm{~h} 07 \mathrm{~m}$ & $07 / 06 / 91$ & 180 & 0.97 & $\mathrm{P}$ & 20 \\
\hline & $R$ & h062 & id & 90 & 0.60 & $\mathrm{~F}$ & 20 \\
\hline
\end{tabular}

\subsection{The CFHT observations}

The CCD camera at the Cassegrain focus was used in the runs of December 1989 and April 1990. It gives a scale of $0.107 \mathrm{arcsec} / \mathrm{pixel}$, with a good sampling even at the best seeing. For the two runs of 1991, the so called HRCam was used at the Prime focus: it compensates for telescope guiding errors using an auxiliary star in the frame field, with some improvement of the seeing. The image scale is practically the same as for the Cassegrain camera. Since the effective entrance aperture for HRCam is reduced to $120 \mathrm{~cm}$, the $S / N$ is much less good, at equal exposure time, than with the original Cassegrain camera.

The used frames are nearly all of sub-arcsec resolution. A small statistics of mesured stellar $F W H M$ is given in Table 4 . The overall means are $0.72 \operatorname{arcsec}$ in $R$ and 0.86 in 
Table 3. List of Pic du Midi frames used in this work. See previous table for explanations

\begin{tabular}{llllllll}
\hline NGC & $F$ & File & Date & Exp & $W$ & $S / N$ & $R$ \\
\hline 3156 & $B$ & $\mathrm{~d} 201$ & $20 / 03 / 90$ & 900 & 1.13 & $\mathrm{P}$ & 35 \\
& $R$ & $\mathrm{~d} 201$ & id & 300 & 0.98 & $\mathrm{P}$ & 35 \\
3193 & $B$ & $\mathrm{~d} 201$ & id & 600 & 1.16 & $\mathrm{P}$ & 30 \\
& $R$ & $\mathrm{~d} 201$ & id & 180 & 0.82 & $\mathrm{P}$ & 30 \\
3605 & $B$ & $\mathrm{~d} 201$ & id & 600 & 1.05 & $\mathrm{P}$ & 20 \\
& $R$ & $\mathrm{~d} 201$ & id & 180 & 1.08 & $\mathrm{P}$ & 20 \\
3608 & $B$ & $\mathrm{~d} 202$ & id & 600 & 0.82 & $\mathrm{P}$ & 35 \\
& $R$ & $\mathrm{~d} 201$ & id & 180 & 0.83 & $\mathrm{P}$ & 35 \\
3640 & $B$ & $\mathrm{~d} 211$ & id & 600 & - & $\mathrm{P}$ & 35 \\
& $R$ & $\mathrm{~d} 211$ & id & 180 & - & $\mathrm{P}$ & 35 \\
3872 & $B$ & $\mathrm{~d} 212$ & id & 600 & 1.32 & $\mathrm{P}$ & 30 \\
& $R$ & $\mathrm{~d} 211$ & id & 180 & 0.92 & $\mathrm{P}$ & 30 \\
\hline
\end{tabular}

Table 4. Statistics of measured stellar FWHM's at the CFHT. Night: date of night beginning in Hawai time. $N$ : number of used observations for each filter. $W_{B}$ : mean $F W H M$ in $B$ (arcsec). $\sigma_{B}$ : standard deviation. $W_{R}$ : mean $F W H M$ in $R . \sigma_{R}$ : standard deviation

\begin{tabular}{llllll}
\hline Night & $N$ & $W_{B}$ & $\sigma_{B}$ & $W_{R}$ & $\sigma_{R}$ \\
\hline $05 / 12 / 89$ & 6 & 1.04 & 0.11 & 0.94 & 0.15 \\
$06 / 12 / 89$ & 8 & 0.79 & 0.10 & 0.60 & 0.05 \\
$04 / 04 / 90$ & 7 & 0.89 & 0.18 & 0.76 & 0.10 \\
$19 / 04 / 91$ & 1 & 0.77 & - & 0.61 & - \\
$20 / 04 / 91$ & 7 & 0.83 & 0.08 & 0.77 & 0.09 \\
$21 / 04 / 91$ & 3 & 0.69 & 0.03 & 0.57 & 0.08 \\
$06 / 06 / 91$ & 7 & 0.81 & 0.15 & 0.65 & 0.10 \\
\hline
\end{tabular}

$B$. On the other hand, the usable field is $641 \times 1011$ pixels, or only $68 \times 108$ arcsec, so that the determination of the sky level is rather crude. In order to get a star suitable for PSF measurement within the field, it was often necessary to position the galaxy well away from the frame centre, with further reduction of the measurable range. Due to specific technical constraints this difficulty is more acute with the HRCam.

\subsection{The $T B L$ observations}

The observations for 6 galaxies are from a single good night (see Table 3). The camera, at the then used focal reducer, gives a scale of $0.315 \mathrm{arcsec} / \mathrm{pixel}$, with adequate sampling for the encountered seeing. For the frames under study, the average stellar $F W H M$ are 0.92 arcsec in $R$ with $\sigma=0.08$ and 1.13 in $B$ with $\sigma=0.20$. Their field is $107 \times 174$ arcsec.

\section{Data analysis}

\subsection{Preliminaries}

The data analysis involves the following preliminary steps:

1. The usual correction of the frames for instrumental effects were made by the observers, following the routines in use at each observatory.

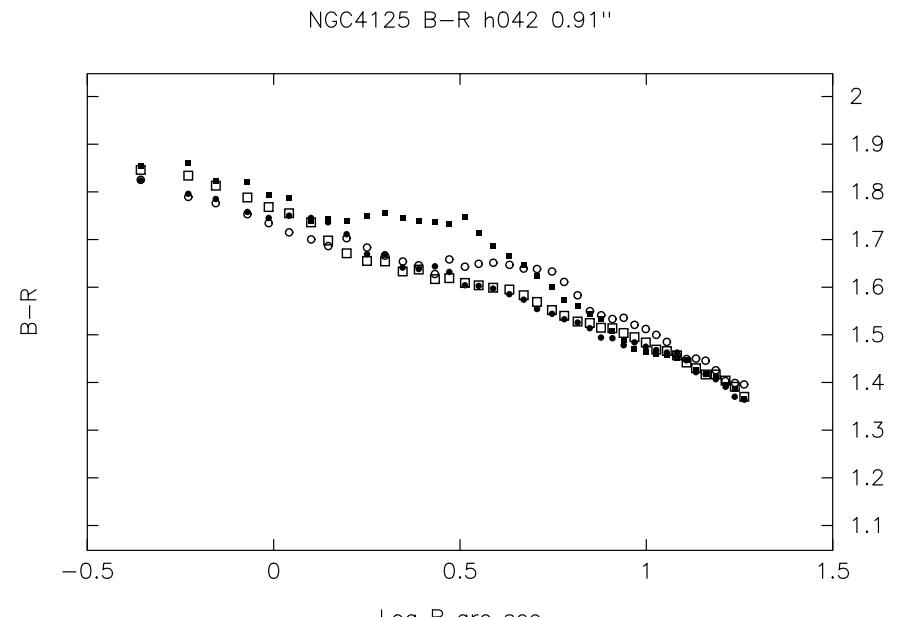

Fig. 1. Sample of a measured radial $B-R$ distribution, i.e. for NGC 4125. Open circles: eastwards maj $A$. Filled circles: westwards $\operatorname{maj} A$. Open squares: southwards $\min A$. Filled squares: northwards $\min A$. The differences are due to an important dust pattern, the data being smoothed by averaging along arcs of isophotes as explained in the text. The upper label gives the code of the $B$-frame and the corresponding seeing $F W H M$

NGC4125 B-R h042 0.91"

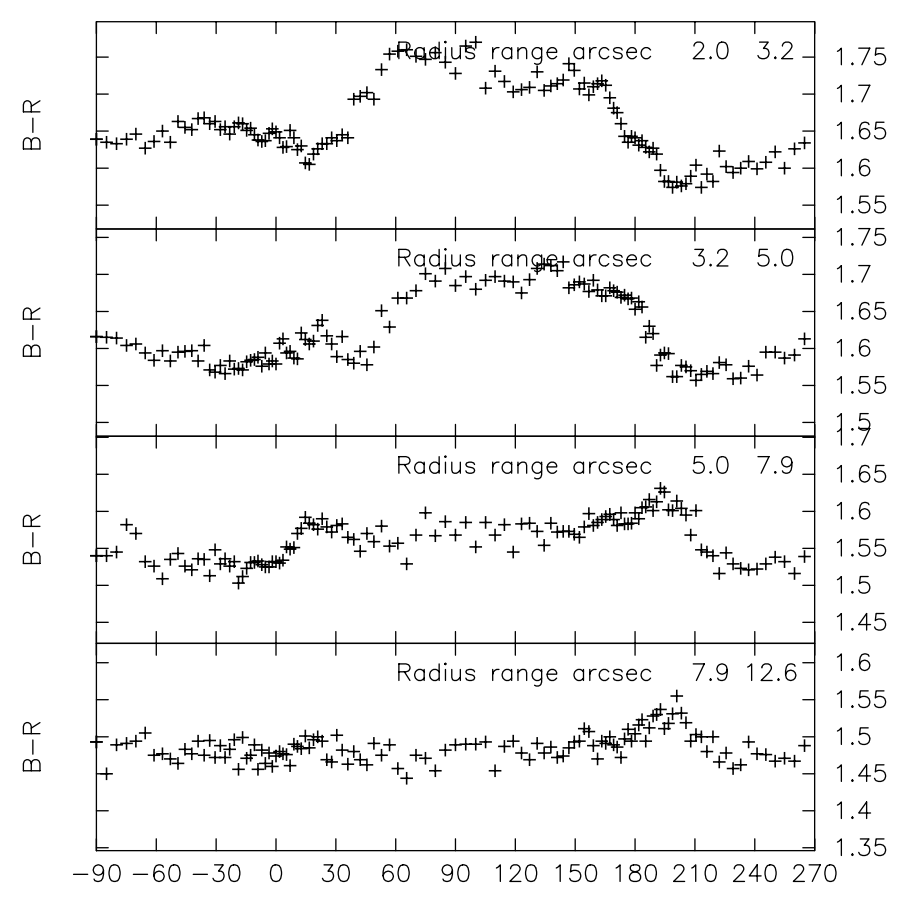

Fig. 2. Sample of a measured azimuthal $B-R$ distribution, i.e. for NGC 4125. Four successive "rings" (as defined in 3.2 .1 ), limited with the isophotes of radii 2.0, 3.2, 5.0, 7.9 and 12.6 arcsec are measured. Abscissae: PA along the isophotes counted counterclokwise from one of the maj $A$, here the one pointing westwards. Ordinate: $B-R$, the scale for each ring being adjusted as needed. The large variations are due to the dust pattern. The upper label gives the code of the $B$ frame and the corresponding seeing $F W H M$ 


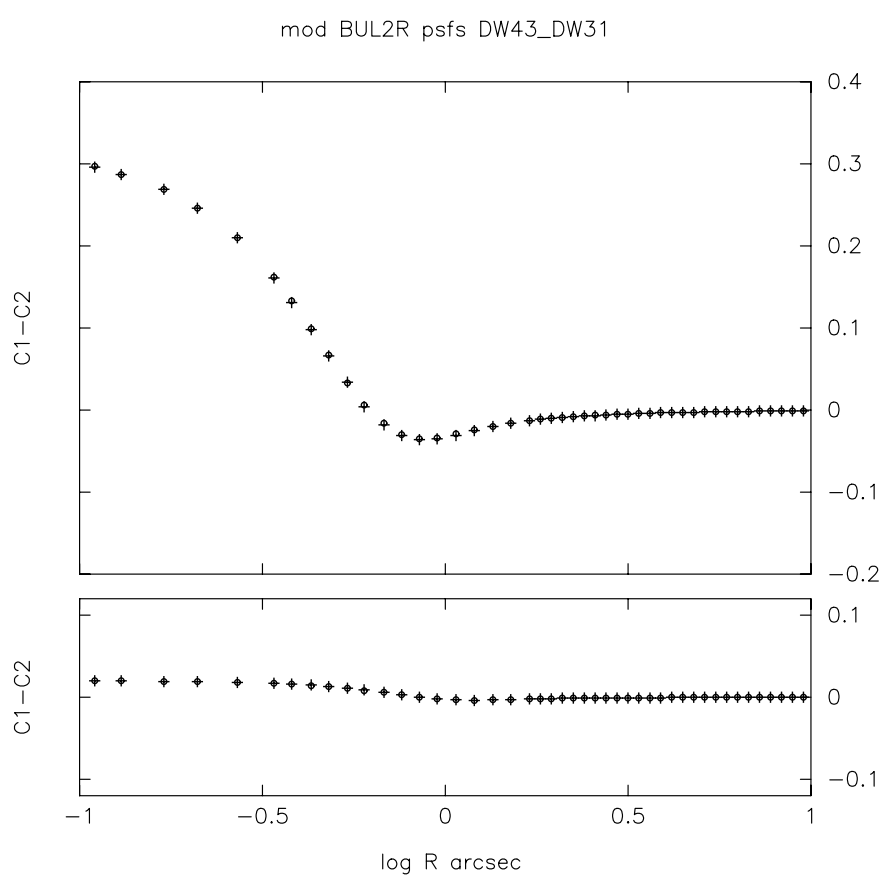

Fig. 3. Experiment showing a pseudo-colour profile induced by differential seeing, and its approximate correction. Abscissae: $\log$ of isophotal mean radius $r$ in arcsec. Ordinates: Colour in magnitude, with circles for the $\operatorname{maj} A$ and crosses for the $\min A$. The model galaxy is a circular $r^{1 / 4}$ bulge (slightly modified), of $F W H M=0.60$ arcsec. The PSF's are sum of Gaussians. Sharper one DW31: $F W H M=0.51$ arcsec with faint wings. Broader one DW43: $F W H M=0.79$ arcsec with strong wings. Upper curve: Colour profile for convolved frames of the model. Lower curve: Colour profile after PSF matching with a single circular gausian (case CG1R). The correction is quite successful

2. The preparation of each frame for measurement was made in the ESO-MIDAS environment, using a procedure summarized by Giudicelli \& Michard (1993). It involves the elimination of significant parasitic objects, the extraction of a suitable stellar image (if any) for the derivation of the PSF, the evaluation of the sky background, the calibration by comparison with the results of aperture photometry (see Sect. 3.3 below); a cosmetic treatment against cosmic ray peaks and anomalous negative pixels, and reduction of the frame to the field deemed necessary. If the $S / N$ ratio is adequate for the derivation of $2 \mathrm{D}$ colour maps, the above treatment may be preceded or completed by the mutual alignement of the frames against the one taken as geometrical reference, possibly using the images of stars to improve the alignment. The output of these preparations are "clean" frames for the galaxy and the PSF, with a number of useful parameters available in the frames "descriptors".

3. Since the colours will be measured along arcs of isophotes, it is a necessary step to obtain a set of isophotes in tabular form. The contours are described by the well known representation first proposed by

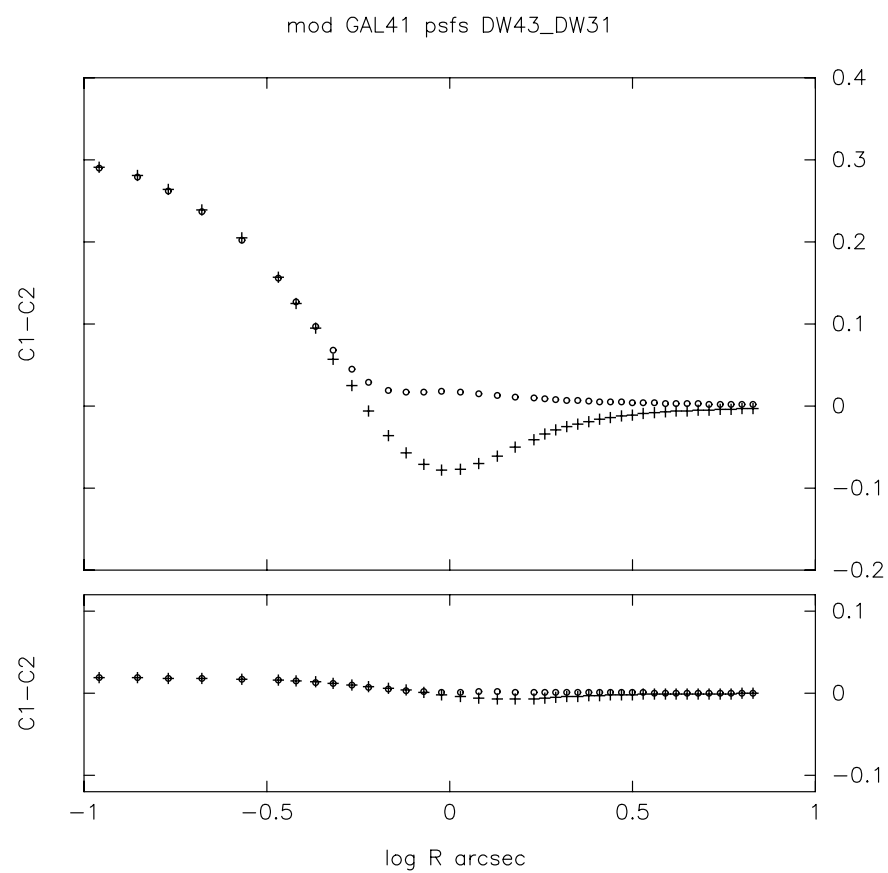

Fig. 4. Experiment showing pseudo-colour profiles induced by differential seeing, and their approximate correction. Abscissae: $\log$ of isophotal mean radius $r$ in arcsec. Ordinates: Colour in magnitude, with circles for the maj $A$ and crosses for the $\min A$. The model galaxy is akin to a flat disky $\mathrm{E}$ or $\mathrm{S} 0$, with a slightly modified $r^{1 / 4}$ bulge of ellipticity $\epsilon=0.5$, plus a disk with $\epsilon=0.74$. The PSF's are sum of Gaussians, i.e. the same as in Fig. 3. Upper curve: Colour profiles for convolved frames of the model. Lower curve: Colour profiles after PSF matching with a single circular gausian (case CG1R). The differential seeing results in a blue light excess on the $\min A$, extending up to $3-4$ times the broad PSF $F W H M$. Again the correction is successful

Carter (1978). For details about our implementation of Carter's representation, see Michard \& Marchal (1994). Only one set of isophotal contours is used to compare two frames and get local colours, but the comparison of Carter's parameters for the two frames may be revealing, as noted for instance by Goodfroij et al. (1994a).

\subsection{Colour measurements}

In previous survey of elliptical galaxies, one was often satisfied with measuring a local reference colour, plus a colour gradient, such as $C_{1}-C_{2}$ and $\mathrm{d}\left(C_{1}-C_{2}\right) / \mathrm{d} \log r$, where $r$ is the equivalent isophotal radius defined above. In general, however, two parameters are far from sufficient to describe the colour distribution in an early-type galaxy. There is no a priori physical reason for the logarithmic colour gradient to remain constant throughout the measured range of radii: it does not, even for a "pure spheroid", if one is able to extend the measurements, either near the centre or outside the central body of the galaxy. There are also significant differences between the 

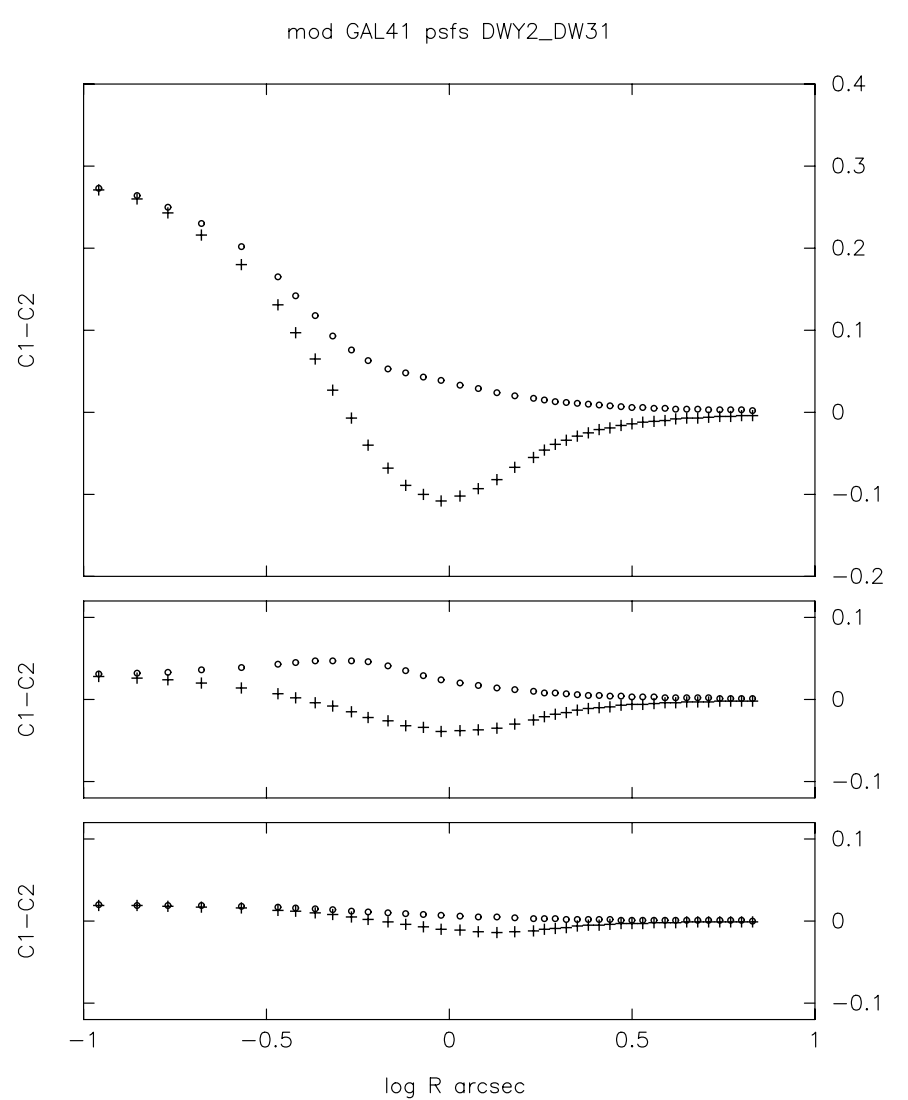

Fig. 5. Experiment showing pseudo-colour profiles induced by differential seeing, and their approximate correction. Abscissae: $\log$ of isophotal mean radius $r$ in arcsec. Ordinates: Colour in magnitude, with circles for the maj $A$ and crosses for the $\min A$. The model galaxy is the flat object considered in Fig. 4. The sharper PSF is the same as in the previous figures, but now the broader PSF DWY2 has extended wings, elongated in the direction of the $\min A$ of the galaxy. $F W H M$ 's: 0.67 arcsec along PSF $\min A ; 0.82$ arcsec along PSF maj $A$. Upper curve: Colour profiles for convolved frames of the model. Intermediate curve: Colour profiles after PSF matching with the best circular Gaussian. Lower curve: Colour profiles after PSF matching with the best elongated Gaussian. The correction whith a circular Gaussian is now unsufficient, but it is successful with a properly elongated Gaussian

gradients in the disk and spheroid of S0's (and diE?), which translate into differences between the major and minor axis gradients for inclined objects. The presence of dust leads to various appearances: local features can sometimes be avoided in order to get more significant colour gradients. Dust layers in disks lead to minor axis asymmetries in light and colours (MS93), while diffuse dust in spheroids will modify apparent light and colour gradients (see the calculations by Witt et al. 1992). It is therefore useful to provide colour data at the relevant level of details, the ultimate being quantitative $2 \mathrm{D}$ colour maps.

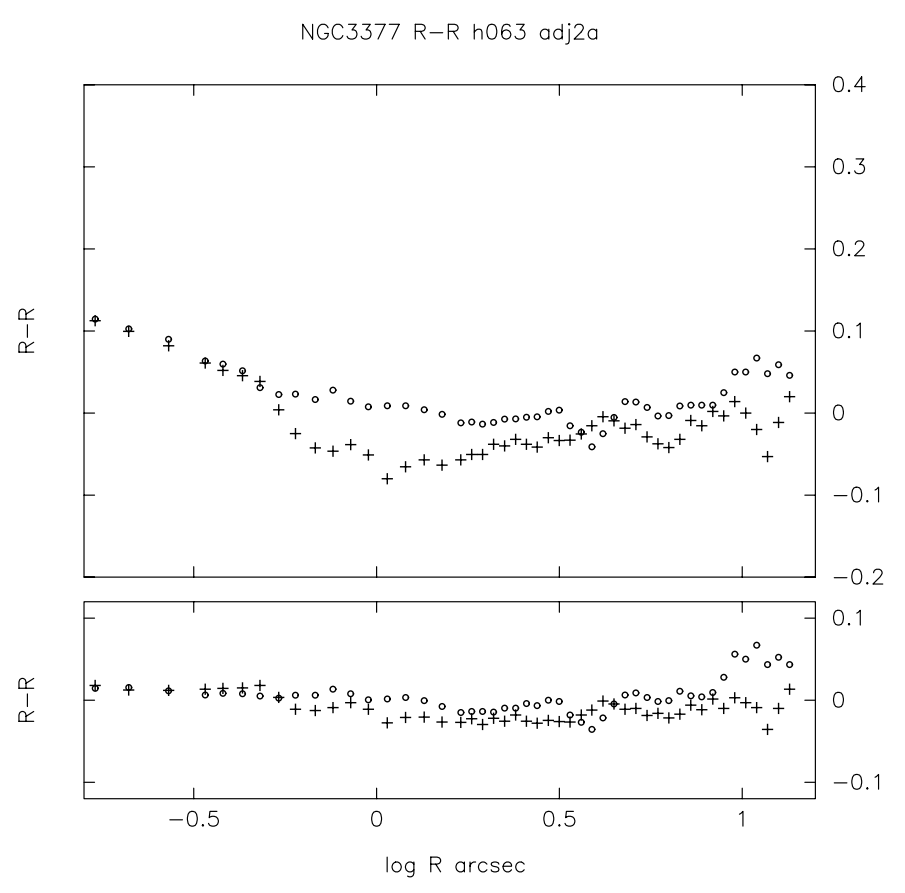

Fig. 6. Experimental correction of the colour profile of a real galaxy, i.e. NGC 3377 for the artefacts of differential seeing. Here are considered two frames taken in the same $R$ passband with measured PSF's of 0.59 and $0.66 \operatorname{arcsec} F W H M$. Abscissae: $\log$ of isophotal mean radius $r$ in $\operatorname{arcsec}$. Ordinates: Colour in magnitude, with circles for the maj $A$ and crosses for the $\min A$. The uncorrected $R-R$ colour (upper graph) shows a central red peak and $\min A$ blueing as for the model of Fig. 4. After correction (lower graph) the $R-R$ colour becomes flat except for noise fluctuations

\subsection{1. $1 \mathrm{D}$ colour measurements}

To provide a good insight into the colour properties of a given object without necessarily ressorting to the $2 \mathrm{D}$ colour maps, we have chosen to measure both the radial and azimuthal colour distributions. We use ad hoc computer programmes, involving as input the two frames to be compared and the table of isophotal contours for one of these: the set of contours is used to locate corresponding points in the two frames. Note that for this particular purpose "symmetrized" contours are used, retaining only the even cosine harmonics of their representation.

To get the radial distributions, local colours are averaged along arcs of the tabulated isophotes, of length 45 degrees in the eccentric anomaly $\omega$ of Carter's Reference Ellipse. Both major and minor axes, and both halves of the two axes, are measured separately. Note that, since the isophotes and isochromes are very much alike in E-S0 galaxies, averaging the colours along moderate intervals in $\omega$ of the isophotal contours, is a technically justified way to improve the $S / N$ ratio.

To get the azimuthal distribution, local colours are averaged inside two concentric isophotes sufficiently apart to improve the $S / N$ ratio, while the azimuthal resolution is kept to 120 points, or 3 degrees in $\omega$. Note that evenly 


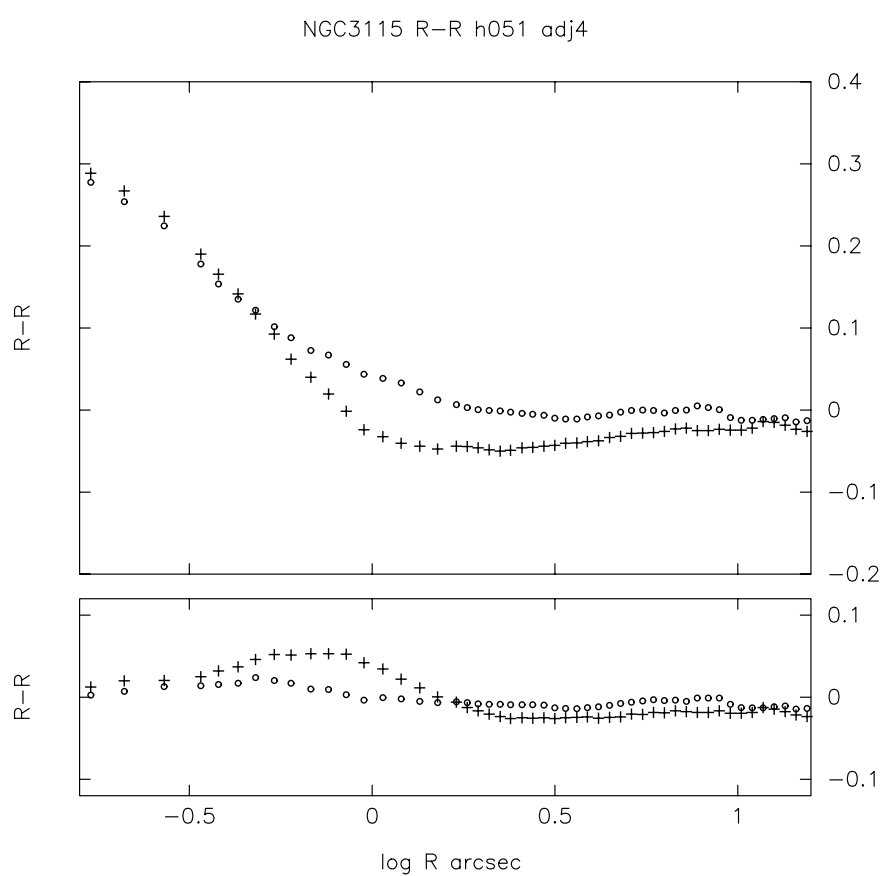

Fig. 7. Experimental correction of the colour profile of a real galaxy, i.e. NGC 3115 for the artefacts of differential seeing. Here are considered two frames taken in the same $R$ passband with measured PSF's of 0.55 and 0.91 arcsec FWHM. Abscissae: $\log$ of isophotal mean radius $r$ in arcsec. Ordinates: Colour in magnitude, with circles for the maj $A$ and crosses for the $\min A$. Due to widely different PSF's, the uncorrected $R-R$ colour (upper graph) shows a strong central red peak and $\min A$ blueing as for the model of Fig. 4. After correction (lower graph) the $R-R$ colour becomes much flatter, except for a local slight bump on the $\min A$

distant points in $\omega$ are not so in position angle from the galaxian centre: they are indeed more closely packed near the tips of the major axis. Suitable software is also available to get the azimuthal colour distribution as a function of the PA itself.

Sample outputs of the above measurements are presented in Figs. 1 and 2 for NGC 4125, a galaxy with a strong dust pattern and an exceptionally large colour gradient. Then the radial $B-R$ profiles are quite different for the 4 mesured arcs, although averaging in rather large azimuthal domains reduces such differences. For the azimuthal profiles the averaging is performed in radial domains as noted in the figure labels, and more detail of the true $B-R$ distributions are kept. A $2 \mathrm{D}$ map of the $B-R$ distribution for the sample galaxy NGC 4125 is shown in Fig. A14 and should be compared with the 1D graphs: it preserves of course more details, but the 1D graphs are certainly useful to appreciate the significance against noise of some features of the distributions.

\subsection{2. $2 \mathrm{D}$ colour maps}

The above 1D graphs will indicate if the colour distribution follows the SuBr distribution or presents significant

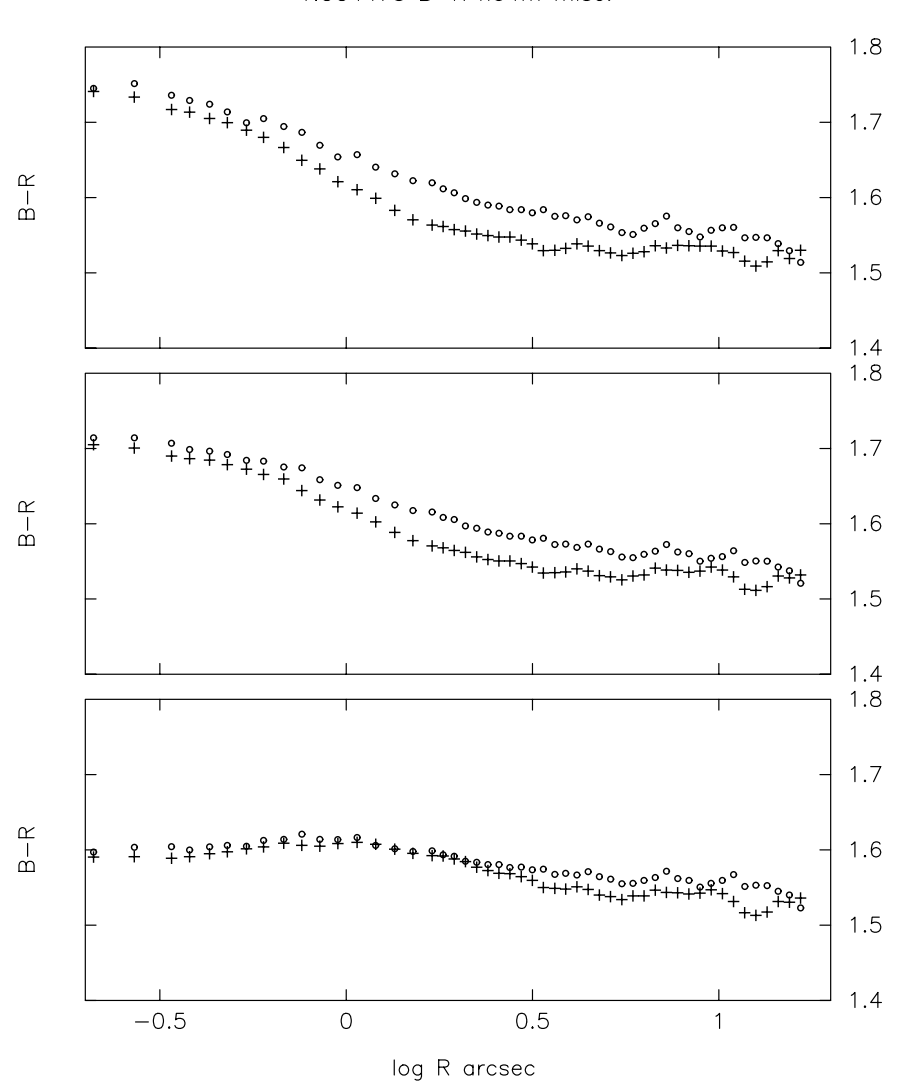

Fig. 8. Corrections to the radial colour distributions for NGC 4473. The frames had seeing FWHM of 1.19 arcsec in $B$ and 1.05 in $R$, poorly measured on a faint star. Abscissae: $\log$ of isophotal mean radius $r$ in arcsec. Ordinates: Colour in magnitude, with circles for the maj $A$ and crosses for the $\min A$. Upper graph: uncorrected results. Intermediate: adopted correction with a Gaussian of $\sigma=0.2$ arcsec. Lower graph: "overcorrection" with $\sigma=0.37$. The central red peak progressively turns out into a blue feature

deviations, due to dust or population variations. In this case it is interesting to consider $2 \mathrm{D}$ colour maps. We have chosen to produce colour maps strictly consistent with the classic astrophysical definition of the colour as a difference of magnitudes. This requires a precise alignment of the two frames, which is not always easily achieved when there are no suitable sets of stars. Very often, one can only put in coincidence the centres of the studied galaxy in the two images... but this centre may be influenced by colour features (and of course by noise).

A number of techniques have been experimented to reduce the noise in colour maps, or more exactly to increase the range of $\mathrm{SuBr}$ where this noise remains acceptable, including the one introduced long ago by Sparks et al. (1985): they replace the redder of the pair of frames by a synthetic image built from a set of isophotal contours. It was concluded however that the improvement was not worth the extra work, since the frames studied here are useful only to measure the innermost range of galaxian colours. 


\subsection{3. $1 \mathrm{D}$ and $2 \mathrm{D}$ asymmetries}

Besides 2D colour maps, we have also considered in some cases, 1D graphs of $\mathrm{SuBr}$ asymmetries as in MS93, and also 2D maps of asymmetries, obtained by comparing a given image with a model of the same, calculated from the corresponding file of isophotal contours: one may use a model with elliptical isophotes, as in van Dokkum \& Franx (1995), or keep the even cosine Carter's coefficients $e_{4}, e_{6}, \ldots$ The model then preserves the diskyness, or boxyness, of the true contours. To get asymmetry maps, we also impose a unique centre and constant orientation to the model isophotes. Such maps have been produced in such cases where the asymmetries were thought to bring useful hints about the dust distribution, in complement to colour maps. By analogy with the findings of MS93, for S0's, it was supposed that dust concentrated in the disk of diE's, might give asymmetries in their inner bulge light: this is indeed the case for a few diE's.

Remark: It should be realized that fitting ellipses produces isophotes that are "interlaced" with the real ones, so that differences between the true image and the model necessarily compensate "dark residuals" (due to extinction?), by nearby "bright residuals". Conversely, the bright residuals due to an embedded disk are compensated by dark residuals that should not be mistaken for absorption markings. In order to get reliable "extinction maps", rather elaborate procedures are necessary, as described by Goudfrooij et al. (1994c).

\subsection{Calibrations and colour corrections}

The frames have been calibrated mostly from the $U B V R I$ aperture photometry by Poulain (1988), and Poulain \& Nieto (1994) where the $R$ band is in Cousins's system. These data are available for 30 objects in the sample. For the others, the catalogues of $U B V$ aperture photometry by Longo \& De Vaucouleurs $(1983,1985)$ were used. The $R$ photometry was obtained from a tight correlation between the observed $B-V$ and $V-R$ from Poulain (1988). For two galaxies, i.e. NGC 3613 and 4649, the available field was too small for calibration with existing aperture photometry: the first was calibrated by fitting to calibrated widefield frames from the Observatoire de Haute-Provence; the second by fitting to the $B$ and $R$ data from Peletier et al. (1990) or PDIDC. As regards the Pic du Midi subset of 6 objects, 3 were calibrated from Poulain's photometry. No significant difference with the CFHT bulk of data is expected.

Poulain's aperture photometry is accurate enough to provide good tests of the sky background. If the sky can be measured with sufficient accuracy from a corner of the frame, the derived photometric zeropoint does not show systematic changes in the various apertures. If not, as it was of course the case for large galaxies, the assumed background value was varied until the test yielded satisfactory results.

The $B-R$ colours collected in Table 6 have been corrected for Galactic reddening and K-effect. It is to be noted that graphical data are not corrected. The $B-V$ colour excesses were derived from the $B$ extinction values given in the Third Reference Catalog of Bright Galaxies, or RC3, by de Vaucouleurs et al. (1991). From Rieke and Lebofsky (1985), we found the galactic colour excess in $B-R$ to be 1.75 larger than $E(B-V)$. For the $\mathrm{K}$ correction the RC3 precepts were followed. and the radial velocities taken from this same source. The proper coefficient was taken from Frei \& Gunn (1994). The work of Fukugita et al. (1995) was also considered.

The resulting CFHT colour system has been compared with the one of PDIDC, using the observed colours at the $r=10$ arcsec contours for 17 objects in common. Note that PDIDC calibrated 30 of their 39 galaxies from Burstein et al. (1987) photometry. A zero point difference for PDIDC-Us of 0.08 is found, with $\sigma=0.04$.

Remark: In the comparison with PDIDC, the galaxy NGC 2768 was neglected: it is found much bluer by PDIDC than here, at $4 \sigma$ of the above mean difference.

\subsection{Errors due to "differential seeing" and their correction}

By differential seeing, we mean the fact that colour measurements involve two frames obtained with different seeing. As the usually encountered PSF's have terribly large effects upon central $\mathrm{SuBr}$ distributions, the difference of the two PSF's will lead to large errors in colours. These errors have been discussed by Vigroux et al. (1988), Franx et al. (1989), Peletier et al. (1990). The later authors derived a cutoff radius for each observation and discarded colours measured inside this limit: in nearly all cases this cutoff is larger than 3 arcsec, although the conditions adopted in its definition cannot be considered as very stringent.

Since we are interested in inner colour distributions, i.e. well inside the cutoff radii of PDIDC, we tried to get approximate corrections for the effects of differential seeing. The essential step of the corrections is to find a function FC, such as the convolution of the sharper of the two PSF's with FC will reproduce the other one. After convolution of the sharper frame with FC the colour distribution will be obtained with the resolution allowed by the worse of the two frames! Another possibility would be to deconvolve the more blurred of the frames by FC, in order to match it with the sharper one. Deconvolution artefacts of the kind described by Michard (1996), might be small enough in this case, because FC is expected to be much narrower than the actual PSF. Of these two possibilities the first one has been used in practice, because it is time saving. 
The derivation of FC is of course dependent upon the availability of a "good" star in the two frames. One can consider several cases, depending upon the $S / N$ of the stellar images and their actual geometry.

1. If the two PSF's can be approximated by Gaussians with circular symmetry, FC is simply another such Gaussian (case C1GR).

2. If the PSF's have important wings, still with circular symmetry, FC can be better approximated by the sum of two Gaussians, intended respectively to match the core and wings of the PSF's (case C2GR).

3 . If the PSF's are elongated, often due to unequal guiding errors in $\alpha$ and $\delta, \mathrm{FC}$ can be better approximated by a Gaussian with different $\sigma$ values in $x$ and $y$ (case C1GXY). Eventually the sum of two Gaussians might be considered (case C2GXY).

These various cases can be implemented by ad hoc MIDAS procedures. Very often however the $S / N$ of available stellar images in our small field frames does not allow much refinements in the derivation of $\mathrm{FC}$, and one has to be satisfied to use the C1GR approximation.

Several experiments have been made to ascertain the effects of differential seeing and the success of the above correction techniques. Part of such experiments were made with model galaxies and model PSF's. The model object, assumed colourless, was convolved with two different PSF's, a sharper one P1 and a poorer P2. The corresponding colour profiles $C_{1}-C_{2}$ were evaluated, and then the above corrections techniques applied. Of course, P1 and P2 are not simple Gaussians, since in this case an exact correction is readily obtained. Figures 3 to 5 present the results of three such experiments.

Other tests were made on real galaxies which have been observed twice in different nights and seeing conditions. Then it is possible to measure the pseudo-colours $B-B$ and $R-R$ resulting from the corresponding frames: this illustrates the effects of differential seeing, and the success of its correction in actual observations, that is in the presence of noise. The Figs. 6 and 7 give examples of these tests, which were applied to 12 pairs of frames. Finally, Fig. 8 shows how the central colour profiles will vary with slight changes in the adopted PSF matching function FC (case C1GR). The central red peak in the "observed" colour of the test object NGC 4473 is lessened by the adopted correction, i.e. with a correcting Gaussian of $\sigma=0.2$ arcsec. It becomes a blue feature with $\sigma=0.4$.

From the tests here described we draw the following conclusions:

1. the errors in peak core colours due to differential seeing may reach several tenths of magnitude, also at the relatively good seeing conditions of the CFHT.

2. such errors, at the level of $0.02 \mathrm{mag}$, extend only up to a radius of twice the $F W H M$ of the PSF (the worse one) for a roundish object and circular PSF's. But the effects are much worse for an elongated PSF "crossing" the $\min A$ of a flattened galaxy (see Fig. 5). In this case the geometry of the inner isochromes may be seriously modified.

3. the errors here discussed may be much reduced by matching the PSF's, as described above. The improvement is limited by difficulties in getting well defined PSF's from the noisy images of faint stars.

\subsection{Comparison of results for multiply observed objects}

For several of the sample galaxies, more than one frame of suitable $S / N$ ratio are available in one or both colours (neglecting very short core exposures!). Such multiple observations may be used to evaluate part of the errors involved in the present work. Two different approaches were found useful.

3.5.1. Pseudo-colours from pairs of frames with the same filter

The derivation of pseudo-colours $B-B$ or $R-R$ gives useful information about errors of various origins. The following cases should be distinguished in these experiments:

1. For frames where the galaxy is located at widely different positions within the instrumental field, or taken with different instrumentation, the errors in background level or flat-field trends will be uncorrelated. The calibrations may also differ, if the number of aperture photometry results is not the same for the two frames. It was found that large residuals may occur under these circumstances. It is therefore advisable to derive colours from pairs of frames taken in succession during the same night and without large offsets of the object within the field. This was the usual practice for the observers who collected the presently used material.

2. For frames taken with the same instrument and with the galaxy at nearly the same location on the CCD target, the residuals in pseudo-colours are due essentially to inaccuracies in the PSF matching. Other errors, such as resulting from the background estimate or residual trends in flat-fielding, will be correlated in the treatment of such parent frames. Results for these cases have been considered above (see Figs. 6 and 7).

3.5.2. Comparisons of colour distributions from different pairs of frames

Such comparisons could be achieved for 8 galaxies: two were observed on two consecutive nights, the second with better seeing; four were re-observed with the HRCam in the hope to get better resolution. For the other there was some duplication of the data in a single night, a case of 
Table 5. Comparison of $B-R$ data from multiple observations. Fr: code for the pair of frames, for reference to Table 1. $W_{B}$ : $F W H M$ in B. $W_{R}: F W H M$ in $R . C_{0}: B-R$ at core centre. $C_{1}: B-R$ at radius $r=1$ arcsec. $C_{3}: B-R$ at radius $r=3$ arcsec. $G r$ : logarithmic $B-R$ gradient for $r>3$ arcsec. The colours are here uncorrected. Nt: Notes to Table 3 (a) No star; mean $F W H M$ 's for the night. (b) Galaxy near the edge of the frame to get a star in. (c) HRCam observations

\begin{tabular}{lllllllll}
\hline NGC & Fr & $W_{B}$ & $W_{R}$ & $C_{0}$ & $C_{1}$ & $C_{3}$ & $G r$ & Nt \\
\hline 3377 & h05 & $1.0 ?$ & $0.9 ?$ & 1.57 & 1.49 & 1.46 & -0.07 & (a) \\
id & h06 & 0.77 & 0.58 & 1.57 & 1.53 & 1.48 & -0.12 & (b) \\
\hline 4406 & h04 & 0.67 & 0.74 & 1.60 & 1.58 & 1.56 & -0.02 & - \\
id & h20 & 0.85 & 0.59 & 1.64 & 1.63 & 1.60 & -0.07 & (c) \\
\hline 4472 & h04 & 0.77 & 0.72 & 1.67 & 1.66 & 1.64 & -0.06 & - \\
id & h20 & 0.82 & 0.72 & 1.65 & 1.64 & 1.61 & -0.10 & (c) \\
\hline 4478 & h04 & 0.86 & 0.72 & 1.57 & 1.50 & 1.46 & -0.05 & - \\
id & h07 & 0.62 & 0.50 & 1.54 & 1.49 & 1.45 & -0.02 & (c) \\
\hline 4494 & h04 & $1.2 ?$ & 0.75 & 1.62 & 1.56 & 1.44 & -0.11 & - \\
id & h20 & 0.89 & 0.79 & 1.66 & 1.57 & 1.48 & -0.07 & (c) \\
\hline
\end{tabular}

limited interest because the differences are due mainly to errors in PSF matching, already discussed above. Here are discussed only the cases where the errors in the $B-R$ distributions are largely independent, except for the errors of calibration. Table 5 presents the results of these comparisons, using ad hoc parameters.

The results of the present experiments are summarized below, both from pseudo-colours and from multiple colour observations:

1. Errors due to imperfect PSF matching are restricted to a radius roughly equal to the worse PSF $F W H M$. An estimate of random errors upon the central core colour is 0.03 . For strongly flattened objects errors upon the $\min A$ colour profile may occur at the same amplitude (see the case of NGC 3115, in Fig. 4).

2. Spurious colour patches at an amplitude of 0.03 may occur due to unsufficient $S / N$.

3. Rather large errors may develop near the limits of the available field. This is due to poor background estimates: these may be worse than in classical observations, where the sky light is effectively registered on the frame due to adequate field of view and generous exposure. As a result the logarithmic gradients of $B-R$ are quite uncertain. The experiment summarized in Table 5 point to a mean difference of 0.05 between two measures of the gradient for the same object! Similarly the mean difference between two measures of the colour at $r_{\mathrm{e}} / 2.5$ is 0.04 . The mean errors upon a single measurement will be slightly smaller.

\subsection{Classification of central $\mathrm{SuBr}$ profiles}

It has been shown by Nieto et al. (1991a), and more recently by Jaffe et al. (1994) and Lauer et al. (1995), that the "cores" of E-galaxies can be sorted out in two types, here termed flat topped core and sharp peak, or respectively $f t c$ and $s h p$. This corresponds to Type I and Type II in the notation of Jaffe et al., or "core-like profile" against "power-law profile" in Lauer et al. wording.

In view of a comparison of central colour profiles with the types of central $\mathrm{SuBr}$ profiles, it was necessary to supplement the lists of "core" types available from the quoted papers. For this purpose, the $R$ frames were deconvolved by Lucy's technique, as implemented in the MIDAS software, using 27 iterations. Then three parameters were examined: change of peak $\mathrm{SuBr}$ between the original and deconvolved frames, or equivalently the ratio of the $F W H M$ 's in the original and deconvolved frames, and finally the $F W H M$ of the deconvolved core. These three parameters indeed show a bimodal distribution. For the first two, this corresponds to the fact that ftc profiles are resolved, or nearly so, at the CFHT resolution, while the $s h p$ profiles remain quite unresolved. The last one is less dependent upon the actual PSF: it would perhaps converge towards an exact galaxian property if the number of iterations was varied in relation with the frame resolution... and if the PSF were perfectly accurate.

The present classification of central profiles, given in Table 6, shows perfect agreement with the one of Faber et al. (1997) for 15 galaxies in common. From the graphs of Byun et al. (1996), there are a few profiles intermediate between the typical "core-like" and "power-law" cases. Similarly our classification gives uncertain results for NGC 4125, 5322, 5576, 5638. Not that the few objects observed at the TBL could not be classified.

\section{Remarkable features in the $B-R$ distributions in E-galaxies}

According to the present study, the colour distributions in E-galaxies may feature the following properties:

1. Colour patterns are made up of localized red patches, and of straight or curved lanes, sometimes forming a complex system. Such local features are unambiguously assigned to dust.

2. The dust in disk phenomenon corresponds to a set of properties in the colour distributions of galaxies containing a disk, and denoting a concentration of dust in this component. The most obvious of such properties is a redder lane along the disk or close to it.

3 . The central red peak stands out in many colour distributions, even after careful matching of the PSF's, as described in Sect. 3. Arguments will be given suggesting that a central dust concentration might be the cause of this feature in many cases, but no conclusive evidence is available.

4. Blue features are scarce enough to be considered as anomalous in E-galaxies.

It should be emphasized that the above listed features are not exclusive of one another, and can very well occur together in a single object. 


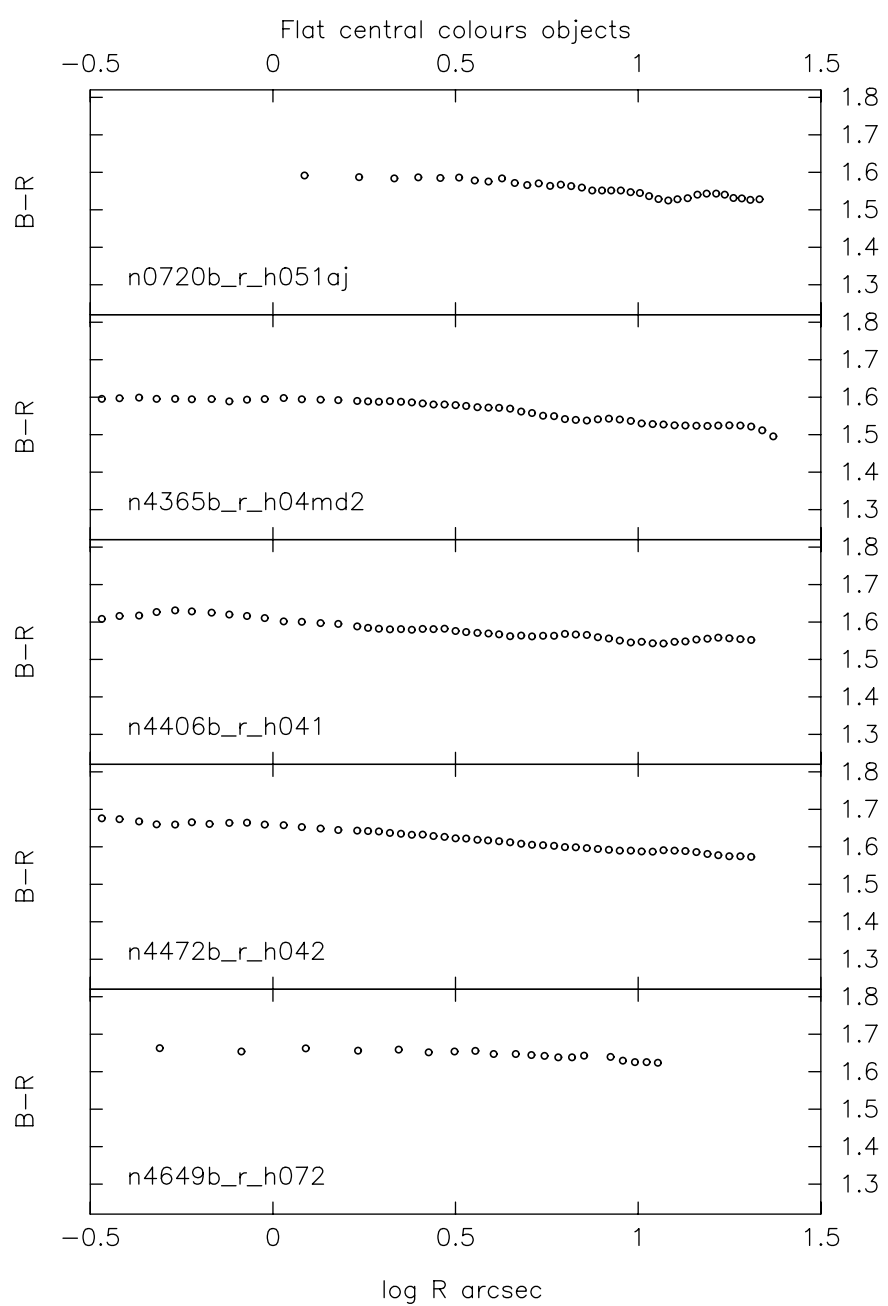

Fig. 9. Sample of objects with flat radial $B-R$ distributions. The label of each graph is the name of the plotted file, which identifies the galaxy. Abscissae: $\log$ of isophotal mean radius $r$ in arcsec. Ordinates: Colour in magnitude. We note that such objects are large galaxies with flat topped cores

\subsection{Local red "colour patterns"}

Local dust features are detected in 16 galaxies out of 42 in our sample, leaving aside the two anomalous blue objects NGC 3156 and 4742. These formations differ widely in size and contrast, and it was deemed useful, for classification purposes, to introduce a dust pattern importance index (DPII), in a scale of 0 to 3 . The various steps of the $D P I I$ can be defined as follows:

. 0: No evidence of local dust in $B-R$ data,

. 1-: Some evidence for local dust is detected,

. 1: Clear dust pattern around core,

2: Marked dust pattern around core,

. 3: Outstanding dust pattern.

For the indices 2 and 3 , the dust produces visible distortions in the $B$ isophotes.

In Paper I of this series, this index was derived for $67 \mathrm{E}$ galaxies of the Local Supercluster, both from the present sample and from literature data (including many multiple

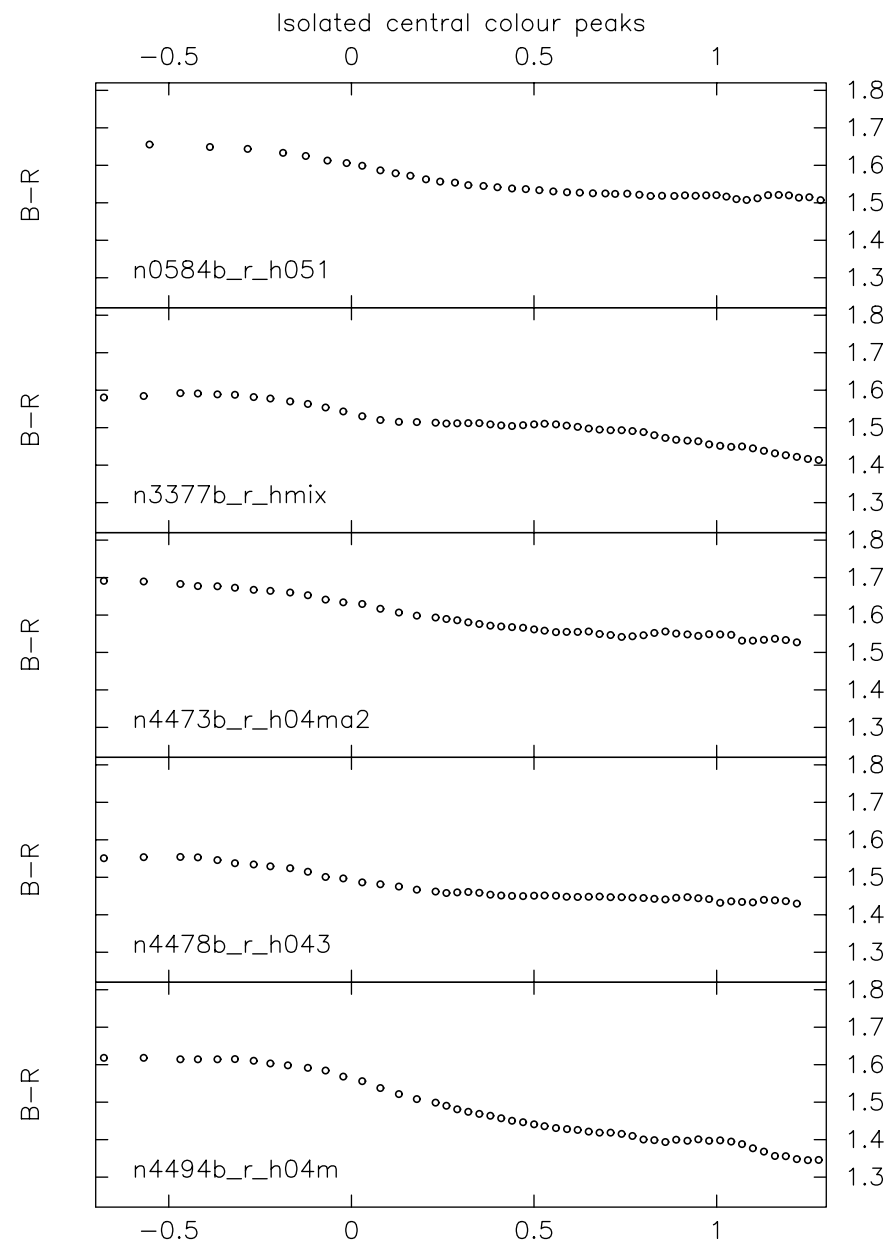

$\log R$ arcsec

Fig. 10. Sample of objects with radial $B-R$ distributions involving a central isolated red peak. The label of each graph is the name of the plotted file, which identifies the galaxy. Abscissae: log of isophotal mean radius $r$ in arcsec. Ordinates: Colour in magnitude. We note that such objects also have a sharply peaked central light distribution

observations), and a statistical discussion was given, relating the DPII to the morphological subclasses, diE, unE and boE, and to other suggestive groupings of objects.

The colour patterns in E's are centrally located, and the galaxian centre is usually the reddest point in the distribution. The pattern of NGC 2974 is an exception, with the peak colour displaced by some 1.5 arcsec.

The strong dust patterns in E galaxies show a geat variety of mostly irregular geometries. Regular rings, as seen in S0 and Sa galaxies at various inclinations are scarcely encountered. The complex colour pattern of NGC 2974 shows however several such rings.

It is perhaps useful to bring attention to a number of dust patterns of special interest as displayed in the maps of Figs. A1 to A32. Outstanding dust patterns $(D P I I=3)$ occur in NGC 1052, 2768, 2974, 4125 and 4374. The case $D P I I=2$ is represented only by NGC 5831 . Is it a subjective trend of our selection or the result of a gap in the real 
NGC3156 B-R d201 1.13"

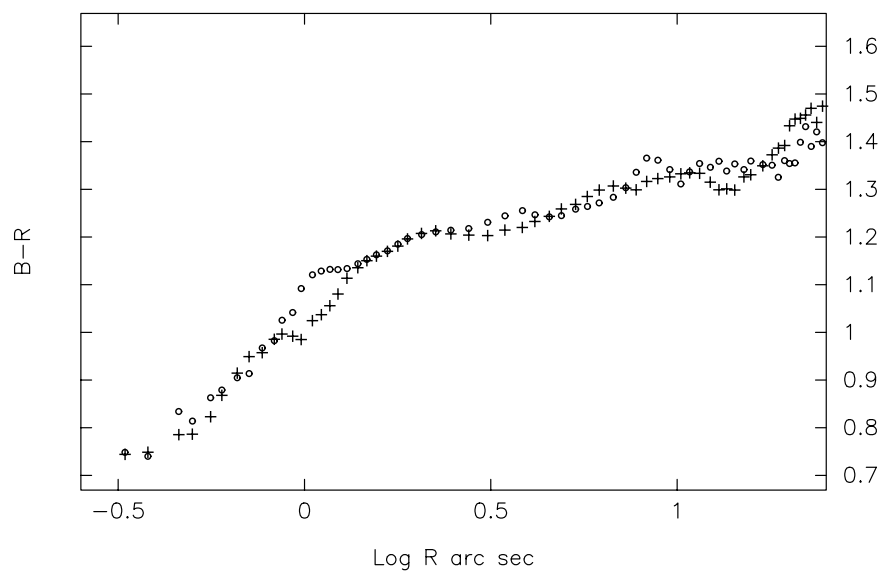

Fig. 11. Radial distribution of $B-R$ for NGC 3156. Abscissae: $\log$ of isophotal mean radius $r$ in arcsec. Ordinates: Uncorrected colour in magnitude. Circles: maj $A$; crosses: $\min A$. This anomalous galaxy contains a centrally located very blue region and also some dust

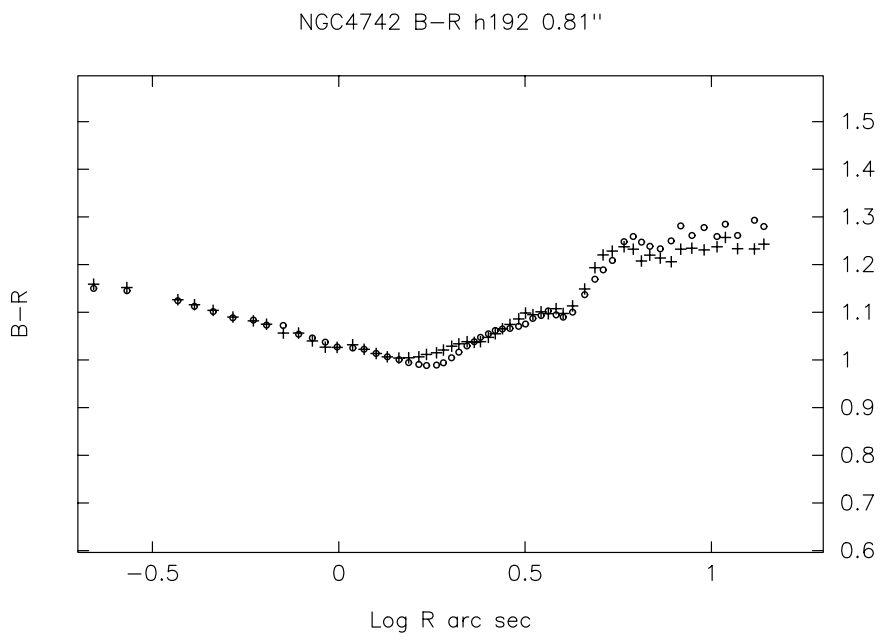

Fig. 12. Radial distribution of $B-R$ for NGC 4742. Abscissae: $\log$ of isophotal mean radius $r$ in arcsec. Ordinates: Uncorrected colour in magnitude. Circles: $\operatorname{maj} A$; crosses: $\min A$. This anomalous galaxy contains a centrally located very blue region with a red peak at the very centre. The available field was quite small so that the colour farther than some 8 arcsec is very uncertain

distribution? The classification of a much larger sample would be necessary to decide.

For $D P I I=1$, we note, among others, NGC 0821, with a short dust lane nearly along the minor axis; and NGC 4660 with a dust lane nearly along the major axis, so that it is also classified among the objects with "dust in disk".

The DPII value 1 - has been given in cases where the isochromes appear to deviate significantly from the isophotes, without a well contrasted colour pattern. Examples are NGC 3377 and 3379. Another case of interest is NGC 4261, where dust is not clearly seen in the $B-R$ distribution, but appears as a small absorption arclet in the $B$ image. This minute feature as been registered with the HST (see Jaffe et al. 1994). There are doubtful cases, where the 1 - index could become 0 with a better $S / N$ ratio, or conversely. For NGC 4494 we find $D P I I=0$ (no dust), while Goudfrooij et al. (1994b) and van Dokkum \& Franx (1995) see dust near the nucleus of this object.

As noted in Paper I, there is generally no evidence for dust patterns in boE galaxies. We refer to the maps of NGC 4365, 4406, 4472 for examples of giant dustless boE's, and to NGC 4387 and 4478 for minor boE's with rotational support (see Nieto \& Bender 1989). The latter have no colour pattern but a sharp red nucleus (see below).

\section{2. "Dust in disk" phenomenon}

In Paper II of this series, the evidence has been considered for dust concentration near the disk of $\mathrm{E}$ galaxies containing a disk component. This involved the disk of diE's at sufficient inclinations, but also the inner "decoupled" disks seen in both boE's and diE's. This evidence may include the "minor axis asymmetry" in light and colour, described and modelled for S0 galaxies in MS93. A distinct reddening of the major axis in the region where the disk contributes significantly to the $\mathrm{SuBr}$ might be also taken as evidence for "dust in disk", but is somewhat ambiguous, because a similar appearance may result from very different colour gradients between the spheroid and disk of a two-component galaxy.

Looking at the set of maps below, the two above properties appear as a flattening of the isochromes as compared to the isophotes, and eventually their displacement in the minor axis direction, relative to the centre of symmetry of the isophotes. These appearances can be recognized in the graphs pertaining to NGC 0584, 3585, 3610, 4473, 4621, 5322,5845 . The latter two objects have inner decoupled disks, and their HST deconvolved images appear in Lauer et al. (1995).

In the summary data table of Sect. 5, galaxies showing evidence of the "dust in disk" phenomenon are noted with the $d d$ symbol.

\subsection{Red nuclei}

Red nuclei in E-galaxies have been considered by Sparks et al. (1985): they note "an unresolved nucleus 0.3 magnitudes redder than the surrounding galaxy" for NGC 6958 . This description obviously refers to an artefact of differential seeing, according to the experiments described in Sect. 3, and to previous studies referenced there. In the present work, the PSF's of the two frames involved in a colour measurement were equalized, and the red nuclei 
can be objectively studied. The resolution is limited by the worse of the two frames, the $B$ one in general, and the accuracy is estimated to $0.02-0.03$ magnitude at the centre and nearby regions.

The central regions show the following types of colour distributions:

1. nearly flat. This is the case for a few large galaxies, i.e. NGC 0720, 4365, 4406, 4472, 4649, 5846 and perhaps also for the compact dwarf NGC 4486B. Such cases are illustrated in Fig. 9. In this situation, the logarithmic colour gradient tends to decrease inwards of a limiting radius of a few arcsec (much less for the dwarf). We note from Table 6 that such objects are in the "flat topped core" ( $f t c$ ) class of central SuBr profiles;

2. isolated red peaks. In this case, illustrated in Fig. 10, the logarithmic colour gradient increases inwards of a break radius $r_{\mathrm{b}}$, with typical values $1.5<r_{\mathrm{b}}<3$ arcsec, for the range of distances and object sizes of our sample. The amplitude of the red nucleus is parametrized by the difference $\Delta C_{0,3}$ of the $B-R$ colours at the centre and at the radius $r=3$ arcsec. Objects with isolated red peaks of large $\Delta C_{0,3}$, notably the examples of Fig. 10, usually belong to the "sharp peak" ( $(\mathrm{h} p)$ class of central $\mathrm{SuBr}$ sprofiles;

3. embedded red peaks. In this case, the red nucleus is part of a larger colour pattern, and the radius $r_{\mathrm{b}}$ is no more defined. The colour difference $\Delta C_{0,3}$ can still be measured but cannot be looked at as a property of the red nucleus itself. The 1D colour graphs are not of much use in such cases. One example has been given in Figs. 1, 2 for NGC 4125.

It is tempting to speculate that central red peaks may be caused by a concentration of dust near the centre of the object (although population variations might also be partly responsible?). This is probably the case for red peaks embedded in a larger dust pattern. Since minute central dust patterns have been discovered from HST frames one might consider these as a likely cause of the red peaks seen at a lesser resolution.

Remark: There are two atypical cases deviating from the above classification of red nuclei. NGC 0636 exhibits a small blue nucleus at the centre of a normal red nucleus; for NGC 2974, the peak $B-R$ is displaced some 1.5 arcsec from the galaxian centre.

\subsection{Anomalous objects}

In our sample, there are 2 galaxies with a central region much bluer than its surroundings, most probably due to recent star formation.

NGC 3156 is an E5 in the RSA, but has type $T=-2$ in the RC3. From the same catalogue, its colours are very blue, 0.75 in $(B-V)_{\mathrm{e}}$ and 0.29 in $(U-B)_{\mathrm{e}}$. The radial $B-R$ distribution is shown in Fig. 11. This galaxy also shows a small dust arclet.
NGC 4742 is an E4 in the RSA, and has type $T=-5$ in the RC3. The catalogue colours are 0.80 for $(B-V)_{\mathrm{T}}$ and 0.30 for $(U-B)_{\mathrm{T}}$. Colours in the effective radius are not available. The radial $B-R$ distribution is shown in Fig. 12. A sharp red peak occurs at the centre of the object.

\section{Data and correlations}

\subsection{Data description}

The $B-R$ data derived from the Nieto's sample is presented as follows:

1. Table 6 lists global characteristics of the studied galaxies and selected numerical, or coded, colour data. Note that reference isophotal colours in the Table were measured outside dust patterns, as well as feasible, and corrected for galactic extinction and K-effect as indicated above, the adopted corrections in $B-R$ being given in the table.

2. The set of Figs. A1 to A32 shows maps of the $B-R$ isochromes and $B$ isophotes in selected small fields, of generally adequate $S / N$ ratio, near the centre of each object.

3. Maps of $B-R$ in FITS format are available in electronic form, upon request to the author. A few samples are available in the anonymous space of the Nice serveur: access by ftp ftp.obs-nice.fr; directory users/bigfic/anonymous/pub/michard.

\subsection{Comparison with other surveys}

Reference colours Previous surveys by Franx et al. (1989) (FIH), Peletier et al. (1990) (PDIDC) and Goudfrooij et al. (1994) (Gea) contain reference colours measured at the isophotal contour of radius $r_{\mathrm{e}} / 2$. We give in Table 6 a $B-R$ isophotal colour $C_{2}$ measured at $r_{\mathrm{e}} / 2.5$, more easily reached in our small field frames. Attempts to plot colourcolour graphs from these data gave somewhat surprising results, even using the two colours from the same survey. It has been supposed that some sets of data involve much larger errors than indicated by the authors. To get some insight into this question, we compared the various sets of reference colours to the mean colours inside $r_{\mathrm{e}}$ derived from aperture photometry by Poulain (1988) or Poulain \& Nieto (1994), supplemented in a few cases by our own estimates from the interpolation of available aperture photometry. Obviously, the isophotal colours at $r_{\mathrm{e}} / 2$ and the mean colours within $r_{\mathrm{e}}$ are not expected to be strictly equal, but cannot differ very much: the former might be somewhat bluer because the latter are possibly influenced by the central dust patterns. For these comparisons the observed mean colours were corrected using the combined corrections applied in each of the surveys. 
The results of the described tests are as follows:

. $(B-R)_{\mathrm{FIH}}-(B-R)_{\mathrm{e}}^{0}$ :

$N=17 ;$ Mean $=0.276 ; \sigma=0.008$

. $(U-B)_{\mathrm{FIH}}-(U-B)_{\mathrm{e}}^{0}$ :

$N=14 ;$ Mean $=-0.030 ; \sigma=0.042$

. $(B-R)_{\mathrm{PDIDC}}-(B-R)_{\mathrm{e}}^{0}$ :

$N=24 ;$ Mean $=0.041 ; \sigma=0.050$

. $(U-B)_{\mathrm{PDIDC}}-(U-B)_{\mathrm{e}}^{0}$ :

$N=24 ;$ Mean $=-0.009 ; \sigma=0.079$

. $(B-V)_{\mathrm{Gea}}-(B-V)_{\mathrm{e}}^{0}$ :

$N=46 ;$ Mean $=-0.000 ; \sigma=0.036$

. $(V-I)_{\text {Gea }}-(V-I)_{\mathrm{e}}^{0}$ :

$N=39 ;$ Mean $=-0.017 ; \sigma=0.166$

. $(B-R)_{\mathrm{Us}}-(B-R)_{\mathrm{e}}^{0}$ :

$N=41 ;$ Mean $=-0.006 ; \sigma=0.039$.

The following suggestions may be made from the numerical results, supplemented by graphs of the compared quantities:

1. The $B-R$ colours of FIH are not in Cousins's system as stated in their paper. A constant correction is adequate to bring their data into this system. As regards their $U-B$ colours, they generally agree well with the $(U-B)_{\mathrm{e}}^{0}$. In view of the small number of objects, 3 values too blue by 0.1 are enough to explain the mean and $\sigma$ above.

2. The $B-R$ colours of PDIDC are somewhat redder than indicated by the here used aperture photometry. This was also found in a direct comparison with our results. Their $U-B$ colours contain a few very unlikely values: NGC 2768 is found much too red, NGC 4486 and 5638 much too blue (at more than $2 \sigma$ ).

3 . The $B-V$ colours of Gea are in good agreement with the $(B-V)_{\mathrm{e}}^{0}$ values. On the other hand their $V-I$ data are plagued with a number of "impossible" values, either too red (i.e. NGC 720) or too blue (i.e. NGC 3377, $4564,5813)$ by more than $2 \sigma$, that is more than 0.33 .

4. Our $B-R$ at $r_{\mathrm{e}} / 2.5$ are in good agreement with the $(B-R)_{\mathrm{e}}^{0}$, as expected from our calibration sources.

Colour gradients Although the present material is far from ideal, as emphasized before, to measure the small colour gradients in E-type galaxies, we have looked at the correlations between our "outer gradient" G12 (see Table 6) and the results from other surveys. We have also compared the gradients for the two available colours in the PDIDC and Gea surveys. This was done by calculating the impartial correlation between centred variables, eventually rejecting extremely divergent values. The results are as follows:

1. If $x=-\Delta(B-V)_{\mathrm{Gea}} / \Delta \log r$ and $y=-\Delta(B-$ $I)_{\mathrm{Gea}} / \Delta \log r$ we find for 42 objects $(2$ rejected) $y=$ $1.95 x+0.009$ with a coefficient of correlation $\rho=0.75$. Thus the gradients in two colours from these authors are mutually consistent.
2. If $x=-\Delta(B-R)_{\mathrm{PDIDC}} / \Delta \log r$ and $y=-\Delta(U-$ $R)_{\mathrm{PDIDc}} / \Delta \log r$ we find for 37 objects $(2$ rejected) $y=2.20 x+0.036$ with a coefficient of correlation $\rho=0.51$. The correlation between the gradients of PDIDC in the two colours appear "weaker than might be expected" to quote these authors. Note that the two $B-R$ and $U-R$ gradients of FIH are very poorly correlated (with only 14 data points).

3. If $x=-\Delta(B-V)_{\mathrm{Gea}} / \Delta \log r$ and $y=-\Delta(B-$ $R)_{\mathrm{Us}} / \Delta \log r$ we find for 21 objects $y=1.40 x-0.019$ with a coefficient of correlation $\rho=0.60$. Our $B-R$ gradients are reasonably consistent with the $B-V$ gradients of Gea.

4. If $x=-\Delta(B-I)_{\mathrm{Gea}} / \Delta \log r$ and $y=-\Delta(B-$ $R)_{\mathrm{Us}} / \Delta \log r$ we find for 20 objects $y=0.73 x-0.027$ with a coefficient of correlation $\rho=0.82$. Our $B-R$ gradients are again reasonably consistent with the $B-I$ gradients of Gea.

5. If $x=-\Delta(B-R)_{\mathrm{PDIDC}} / \Delta \log r$ and $y=-\Delta(B-$ $R)_{\mathrm{Us}} / \Delta \log r$ we find for 19 objects $y=0.92 x+0.013$ with a coefficient of correlation $\rho=0.41$. Our $B-R$ gradients do not correlate as well with the gradients of PDIDC as with those of Gea.

Dust patterns The mappings of large dust patterns by various authors are generally in reasonable ageement. This is the case when comparing our data with the $V-I$ map of NGC 1052 by Sparks et al. (1985), or the maps of Goudfrooij et al. (1994b), for NGC 2974, 3377, 4125, 4374, 4660 .

Discrepancies arise, when one of the intercompared surveys is of much lesser resolution, or perhaps affected by the effects here described as "differential seeing". For instance, Goudfrooij et al. find a blue inner disk in NGC 3610 and 4473, in contradiction with our data. An interesting case is NGC 4494: for this object $V-I$ maps have been obtained from HST frames by Forbes et al. (1995), and by Carollo et al. (1997). These show a dust ring of subarcsec radius, obviously located in the inner disk, and much stronger on the $\mathrm{W}$ side of the major axis. We have overlooked this feature, although it produces in our $B-R$ map (Fig. A23) a clear $E-W$ colour asymmetry (for this same object, Goudfrooij et al. note a minor axis dust lane). NGC 4494 is an ideal example in favour of our speculation above, that a central accumulation of dust might be responsible for the isolated red nuclei found in a number of objects of our $s h p$ class. From a glance at the $V-I$ radial profiles and $V-I$ images of the above quoted authors, it seems that several other galaxies of the "power-law" class have both nuclear dust and a sharp red peak.

Dust features may well be missed in the present survey due the poor $S / N$ ratio of part of our frames (quoted $\mathrm{P}$ in Table 1). For instance a faint dust pattern is found near 
Table 6. Isophotal colours at selected radii and gradients. $M_{\mathrm{T}}$ : Absolute magnitude in $B$ from Michard \& Marchal (1994), or derived accordingly. $\log r_{\mathrm{e}}$ : Logarithm of the effective isophotal radius in arcsec from same source. $\Delta(B-R)$; Corrections to the observed $B-R$ for galactic extinction and K-effect. CP: ftc for a flat topped core, shp for a sharp peak. $C_{0}$ : Peak central corrected $B-R$ colour. $\Delta C_{0,3}$ colour difference between centre and radius $r=3 \operatorname{arcsec}$. $C_{1}$ : Corrected $B-R$ at $r_{\mathrm{e}} / 10$. It is uncertain, or not measured, if $r_{\mathrm{e}}<10 . C_{2}$ : Corrected $B-R$ at $r_{\mathrm{e}} / 2.5$. It involves an extrapolation for large objects. $G_{12}$ : Outer logarithmic gradient for $r>3$ arcsec. Code: Dust pattern importance index DPII and code dd for "dust in disk". A colon: refers to an uncertain result.

Notes to Table 6: (a) NGC 0636: small blue dot in core: representative core colour interpolated. (b) NGC 2974: peak $B-R$ in patch 1.7 arcsec NW of centre. (c) NGC 3156: Very anomalous colour distribution (see Fig. 11) (d) NGC 4125: Extraordinary gradient (see also Goudfrooij et al. 1994a) (e) NGC 4742: Very anomalous colour distribution (see Fig. 12) (f) NGC 5322: Inner small disk, with some reddening, isolated from main boxy body. (g) NGC 5845: Inner small disk, with some reddening, isolated from main disky body

\begin{tabular}{|c|c|c|c|c|c|c|c|c|c|c|c|c|}
\hline $\mathrm{NGC}$ & Type & $-M_{\mathrm{T}}$ & $\log r_{\mathrm{e}}$ & $\Delta(B-R)$ & $\mathrm{CP}$ & $C_{0}$ & $\Delta C_{0,3}$ & $C_{1}$ & $C_{2}$ & $G_{12}$ & Code & - \\
\hline 0584 & $\mathrm{diE}$ & 19.82 & 1.37 & 0.08 & $\operatorname{shp}$ & 1.57 & 0.11 & 1.47 & 1.43 & -.03 & $0 \mathrm{dd}$ & - \\
\hline 0596 & diEp & 19.66 & 1.43 & 0.07 & $\operatorname{shp}$ & 1.57: & 0.07 & 1.52 & 1.47 & -.04 & $1-$ & - \\
\hline 0636 & diEp & 18.98 & 1.31 & 0.08 & $\operatorname{shp}$ & 1.52 & 0.06: & 1.48 & 1.42 & -.07 & 0 & (a) \\
\hline 0720 & unE & 20.85 & 1.53 & 0.02 & $\mathrm{ftc}$ & 1.62 & 0.02 & 1.60 & 1.56 & -.05 : & 0 & - \\
\hline 0821 & $\operatorname{diE}$ & 20.54 & 1.51 & 0.09 & $\operatorname{shp}$ & 1.63 & 0.05 & 1.59 & 1.54 & -.07 & $1 \mathrm{dd}:$ & - \\
\hline 1052 & $\operatorname{diE}$ & 19.79 & 1.37 & 0.04 & $\operatorname{shp}$ & 1.82 & 0.19 & 1.62 & 1.53 & -.10 : & 3 & - \\
\hline 2768 & $\operatorname{diE}$ & 21.13 & 1.70 & 0.09 & shp & 1.85 & 0.16 & 1.67 & 1.61 & -.11 : & 3 & - \\
\hline 2974 & $\operatorname{diE}$ & 20.41 & 1.38 & 0.07 & shp & 1.63 & 0.09 & 1.55 & 1.52 & -.11 : & 3 & (b) \\
\hline 3156 & SA0 & 17.62 & 1.17 & 0.03 & - & 0.66 & - & 1.12 & 1.24 & +.20 & $1-$ & (c) \\
\hline 3193 & unE & 19.68 & 1.21 & 0.05 & - & 1.55 & 0.07 & 1.50 & 1.47 & -.05 & 0 & - \\
\hline 3377 & $\operatorname{diE}$ & 19.25 & 1.56 & 0.04 & $\operatorname{shp}$ & 1.56 & 0.10 & 1.46 & 1,40 & -.10 & $1-\mathrm{dd}$ & - \\
\hline 3379 & unE & 20.11 & 1.75 & 0.03 & $\mathrm{ftc}$ & 1.62 & 0.05 & 1.55 & 1.52 & -.05 & $1-$ & - \\
\hline 3585 & $\operatorname{diE}$ & 20.05 & 1.30 & 0.14 & shp & 1.57 & 0.06 & 1.51 & 1.46 & -.06 & $0 \mathrm{dd}$ & - \\
\hline 3605 & boE & 17.99 & 0.87 & 0.01 & - & 1.51 & 0.14 & - & 1.38 & $-.10:$ & 0 & - \\
\hline 3608 & boE & 19.63 & 1.56 & 0.02 & ftc: & 1.60 & 0.08 & 1.52 & 1.50 & -.03 & 1- & - \\
\hline 3610 & $\operatorname{diE}$ & 19.06 & 1.13 & 0.02 & shp & 1.56 & 0.10 & 1.48 & 1.43 & -.06 & $0 \mathrm{dd}$ & - \\
\hline 3613 & $\operatorname{diE}$ & 18.93 & 1.33 & 0.03 & $\operatorname{shp}$ & 1.54 & 0.06 & 1.49 & 1.47 & -.03 & 1-: & - \\
\hline 3640 & boE & 19.85 & 1.34 & 0.06 & - & 1.57 & 0.09 & 1.48 & 1.45 & -.04 & 0 & - \\
\hline 3872 & diEp & 20.55 & 1.13 & 0.09 & - & 1.55 & 0.05 & - & 1.50 & $-.01:$ & 0 & - \\
\hline 4125 & $\operatorname{diE}$ & 20.55 & 1.55 & 0.03 & shp & 1.80 & 0.20 & 1.60 & 1.44 & -.25 & 3 & (d) \\
\hline 4261 & boE & 20.98 & 1.59 & 0.03 & $\mathrm{ftc}$ & 1.68 & 0.10 & 1.57 & 1.54 & -.05 & 1- & - \\
\hline 4365 & boE & 20.65 & 2.00 & 0.02 & $\mathrm{ftc}$ & 1.61 & 0.02 & 1.55 & 1.53: & -.03 & 0 & - \\
\hline 4374 & unE & 21.15 & 2.02 & 0.06 & $\mathrm{ftc}$ & 1.79 & 0.18 & 1.56 & 1.53: & $-.04:$ & 3 & - \\
\hline 4387 & boE & 18.90 & 0.96 & 0.04 & $\operatorname{shp}$ & 1.60 & 0.07 & - & 1.53 & -.08 & 0 & - \\
\hline 4406 & boE & 21.44 & 2.20 & 0.04 & $\mathrm{ftc}$ & 1.59 & 0.03 & 1.52 & 1.50: & -.04 & 0 & - \\
\hline 4472 & unE & 21.65 & 2.05 & 0.01 & $\mathrm{ftc}$ & 1.65 & 0.03 & 1.57 & 1.53 & -.06 & 0 & - \\
\hline 4473 & $\operatorname{diE}$ & 19.92 & 1.45 & 0.04 & $\mathrm{ftc}$ & 1.64 & 0.11 & 1.53 & 1.50 & -.05 & $0 \mathrm{dd}$ & - \\
\hline 4478 & boEp & 18.78 & 1.03 & 0.06 & $\operatorname{shp}$ & 1.53 & 0.11 & - & 1.39 & -.04 & 0 & - \\
\hline 4486B & unEp & 17.63 & 0.59 & 0.06 & $\mathrm{ftc}$ & 1.57 & 0.04 & - & 1.53 & -.00 & 0 & - \\
\hline 4494 & unE & 19.57 & 1.56 & 0.04 & $\operatorname{shp}$ & 1.60 & 0.15 & 1.43 & 1.36 & -.09 & $0:$ & - \\
\hline 4551 & boE & 18.06 & 1.13 & 0.08 & shp & 1.60 & 0.13 & - & 1.43 & -.06 & 0 & - \\
\hline 4564 & $\operatorname{diE}$ & 18.99 & 1.25 & 0.05 & $\operatorname{shp}$ & 1.64 & 0.12 & 1.56 & 1.48 & -.15 & $1-\mathrm{dd}$ & - \\
\hline 4621 & $\operatorname{diE}$ & 20.18 & 1.68 & 0.03 & $\operatorname{shp}$ & 1.65 & 0.10 & 1.52 & 1.47: & $-.08:$ & $0 \mathrm{dd}$ & - \\
\hline 4649 & $\mathrm{boE}$ & 21.10 & 1.85 & 0.03 & $\mathrm{ftc}$ & 1.63 & 0.00 & 1.61 & 1.58 & $-.04:$ & 0 & - \\
\hline 4660 & $\operatorname{diE}$ & 18.55 & 1.09 & 0.01 & $\operatorname{shp}$ & 1.65 & 0.09 & - & 1.51 & -.08 & $1 \mathrm{dd}$ & - \\
\hline 4742 & SA0: & 18.15 & 0.65 & 0.03 & - & 1.14 & - & 1.07 & 1.00 & +.40 & 0 & (e) \\
\hline 5322 & boE & 20.44 & 1.54 & 0.03 & shp: & 1.55 & 0.13 & 1.41 & 1.36 & -.09 & $0 \mathrm{dd}$ & (f) \\
\hline 5576 & boEp & 20.16 & 1.55 & 0.03 & $\operatorname{shp}$ & 1.50 & 0.11 & 1.38 & 1.34 & -.06 & 0 & - \\
\hline 5638 & unE & 19.50 & 1.35 & 0.04 & shp: & 1.56 & 0.05 & 1.52 & 1.48 & -.06 & 0 & - \\
\hline 5813 & unE & 20.66 & 1.59 & 0.09 & $\mathrm{ftc}$ & 1.62 & 0.09 & 1.52 & 1.47 & -.10 & 1 & - \\
\hline 5831 & $\mathrm{diE}$ & 19.62 & 1.33 & 0.08 & shp & 1.60 & 0.11 & 1.51 & 1.46 & -.10 & 2 & - \\
\hline 5845 & $\mathrm{diE}$ & 19.58 & 0.57 & 0.08 & shp & 1.56 & 0.09 & 1.49 & 1.44 & -.09 & $0 \mathrm{dd}$ & (g) \\
\hline 5846 & unE & 21.00 & 1.67 & 0.08 & $\mathrm{ftc}$ & 1.67 & 0.03 & 1.61 & 1.54: & -.06 & 0 & - \\
\hline $5846 \mathrm{~A}$ & unE & 17.40 & 0.35 & 0.08 & $\operatorname{shp}$ & 1.65 & 0.05 & - & 1.59: & $-.04:$ & 0 & - \\
\hline
\end{tabular}


the center of NGC 5846 by Goudfrooij \& Trinchieri (1998), which was not detected from our low exposure frames.

\section{Concluding remarks}

This paper has been devoted to the description of techniques and the presentation of data about the $B-R$ colour distributions in E-type galaxies, as measured from rather good resolution frames. The scientific results have been given in the three previous paper of this series and again summarized in the Introduction.

Therefore the concluding remarks will be devoted to technical matters.

1. It has been proven that colour measurements can be extended inside the "seeing" degraded regions of galaxian images, if a suitable treatment is applied to equalize the different PSF's in the two frames involved in any colour. This treatment may be a convolution of the "best" frame by a correcting function, or eventually the deconvolution of the "worse" by this same function. This procedure is successful only if the two frames have similar "seeing", so that the correcting function is narrow enough, and well measured from a suitable star in the field.

2. The short discussion of available colour surveys of E-type galaxies in the above section, suggests that the data are not in a well defined and unique colour system, and eventually suffer from serious calibration errors for a number of objects. A reconsideration of the data, and perhaps new observations, may be needed to obtain a fully reliable set of colours and their gradients, in the usual optical colours, for a significant sample of E-type objects.

\section{Appendix A:}

This contains maps of the $B-R$ isochromes (full lines) and $B$ isophotes for most of the galaxies in the present sample, at least if the data was of acceptable $S / N$ ratio. A few maps of poor $S / N$ were included if thought to convey some useful information. Since the HRCam has to be rotated to get both the object and the guiding star in the field, the approximate direction of the North is given in the captions. No image reversal occurs, so that the East is always $90^{\circ}$ counterclockwise from the North.

\section{References}

Arnaboldi M., Da Costa G.S., Saha P., 1996, "The Nature of Elliptical Galaxies", 2d. Stromlo Symp., Canberra, 26-30 Aug. 1996, ASP Conf. Ser., 1997

Balcells M., Peletier R.F., 1994, AJ 107, 13

Burstein D., Davies R.L., Dressler A., et al., 1987, ApJS 64, 601

Byun Y., Grillmair C.J., Faber S.M., et al., 1996, AJ 111, 1889
Carollo C.M., Franx M., Illingworth G.D., et al., 1997, ApJ 481,710

Carter D., 1978, MNRAS 182, 797

van Dokkum P.G., Franx M., 1995, AJ 110, 2027

Faber S.M., Tremaine S., Ajhar E.A., et al., 1997, AJ 114, 1771

Franx M., Illingworth G., Heckman T., 1989, AJ 98, 538 (FIH)

Forbes D.A, Franx M., Illingworth G.D., 1995, AJ 109, 1988

Frei Z., Gunn J.E., 1994, AJ 108, 1476

Fukugita M., Shimasaku K., Ichikawa T., 1995, PASP 107, 945

Giudicelli M., Michard R., 1993, Proceedings 5th ESO/STECF Data Analysis Workshop, p. 105

Goudfrooij P., Hansen L., Jorgensen H.E., et al., 1994a, A\&AS 104, 179 (Gea)

Goudfrooij P., Hansen L., Jorgensen H.E., et al., 1994b, A\&AS 105,341

Goudfrooij P., de Jong T., Hansen L., et al., 1994c, MNRAS 271,833

Goudfrooij P., 1995, The Messenger 79, 31

Goudfrooij P., 1996, in "The Nature of Elliptical Galaxies", 2d. Stromlo Symp., Canberra, 26-30 Aug. 1996, ASP Conf. Ser., 1997, Arnaboldi M., Da Costa G.S., Saha P. (eds.)

Goudfrooij P., Trinchieri G., 1998, A\&A 330, 123

Jaffe W., Ford H.C., O'Connell R.W., et al., 1994, AJ 108, 1567

Lauer T.R., Ajhar E.A., Byun Y., et al., 1995, AJ 110, 2622

Longo G., de Vaucouleurs A., 1983, A General Catalogue of Photoelectric Magnitudes and Colours in the $U B V$ System of 3578 Galaxies brighter than the 16th $V$-magnitude, 1936-1982, The Univ. of Texas Publ.

Longo G., de Vaucouleurs A., 1985, Supplement to the General Catalogue of Photoelectric Magnitudes and Colowith circles for the $\operatorname{maj} A$ and crosses for the $\min A$. rs in the $U B V$ System, The Univ. of Texas Publ.

Michard R., Simien F., 1993, A\&A 274, L25 (MS93)

Michard R., 1994, A\&A 288, 401

Michard R., 1996, A\&AS 117, 583

Michard R., Marchal J., 1994, A\&AS 105, 81

Michard R., 1998a, A\&A 334, 453

Michard R., 1998b, A\&A 335, 479

Michard R., 1998c, A\&A 335, 49

Nieto J.L., Bender R., 1989, A\&A 215, 266

Nieto J.L., Bender R., Arnaud J., et al., 1991a, A\&A 244, L25

Nieto J.L., Bender R., Surma P., 1991b, A\&A 244, L37

Peletier R.F., Davies R.L., Illingworth G.D., et al., 1990, AJ 100, 1091 (PDIDC)

Peletier R.F., Balcells M., 1996, AJ 111, 2238

Poulain P., 1988, A\&AS 72, 215

Poulain P., Nieto J.L., 1994, A\&AS 103, 573

Rieke G.H., Lebofsky M.J., 1985, ApJ 288, 618

Silva D.R., Elston R., 1994, ApJ 428, 511

Silva D.R., Wise M.W., 1996, ApJ 457, L15

Silva D.R., Boroson T.A., Thompson I.B., et al., 1989, AJ 98, 131

Sparks W.B., Walf J.V., Thorne D.J., et al., 1985, MNRAS 218, 87

de Vaucouleurs G., de Vaucouleurs A., Corwin H.G.Jr., et al., 1991, Third Reference Catalogue of Bright Galaxies. Springer Verlag, New-York

Vigroux L., Souviron J., Lachieze-Rey M., et al., 1988, A\&AS 73,1

Wise M.W., Silva D.R., 1996, ApJ 461, 155

Witt A., Thronson H.A.jr, Capuano J.M., 1992, ApJ 393, 611 


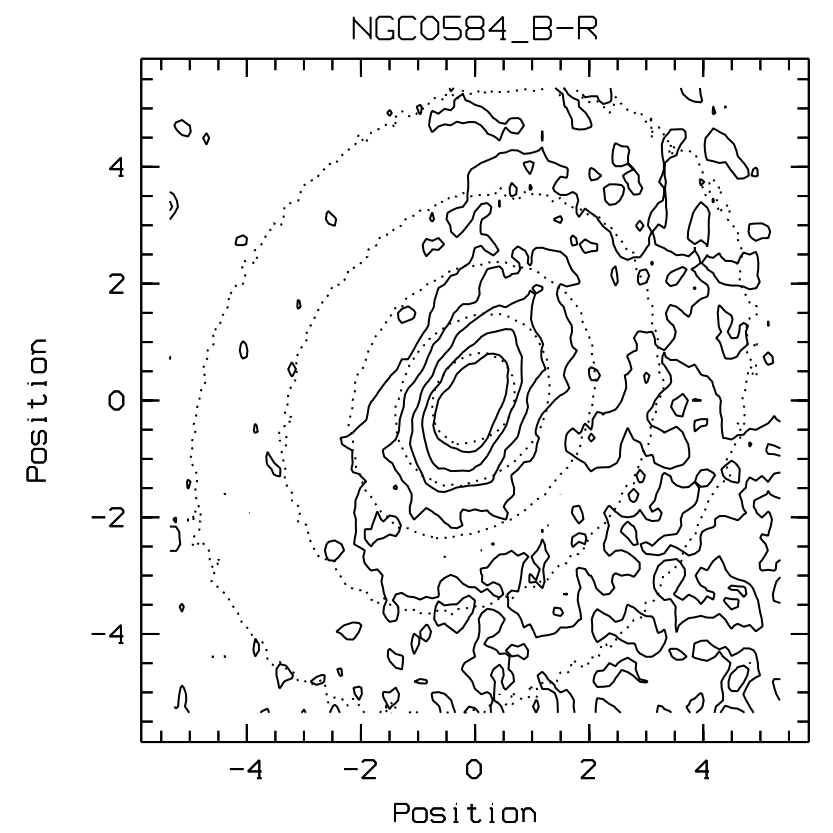

Fig. A1. NGC 0584: diE. Positions in ". $N$ at 9 o'clock. $B-R$ isochromes: $1.475-1.60$ (0.025). $B$ isophotes: $17.0-19.0$ (0.5). The flattening of the isochromes, and their shift along the minor axis, suggest dust concentration in the disk

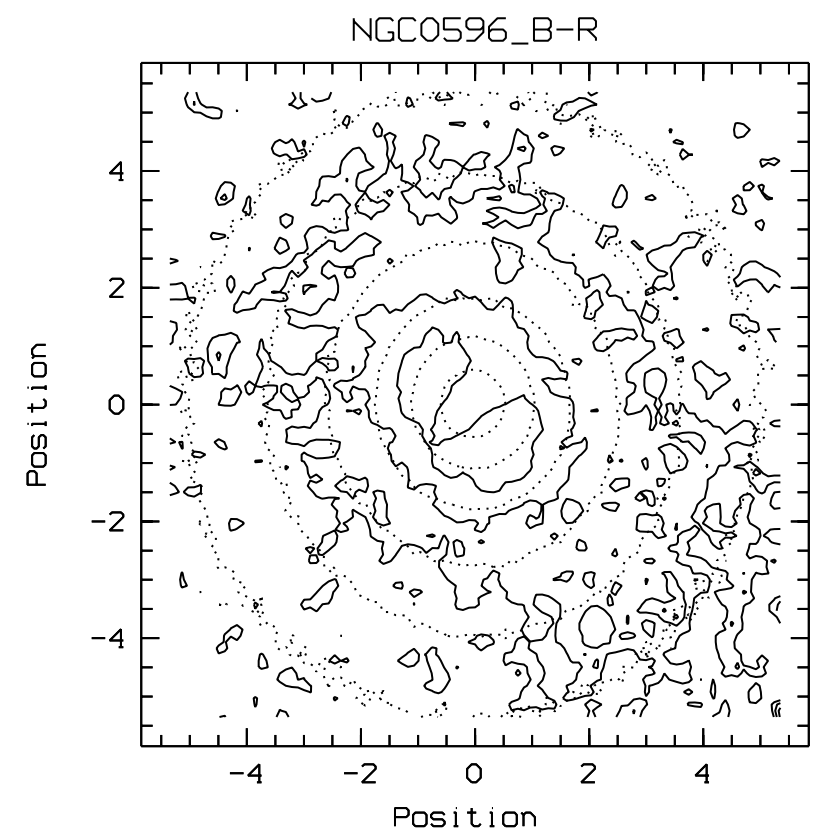

Fig. A2. NGC 0596: diEp. Positions in ". $N$ at 9 o'clock. $B-R$ isochromes: $1.57-1.62(0.025)$. $B$ isophotes: $17.2-19.7$ (0.5). The slightly tilted, low contrast red pattern, suggests only little dust. Possible blue core feature

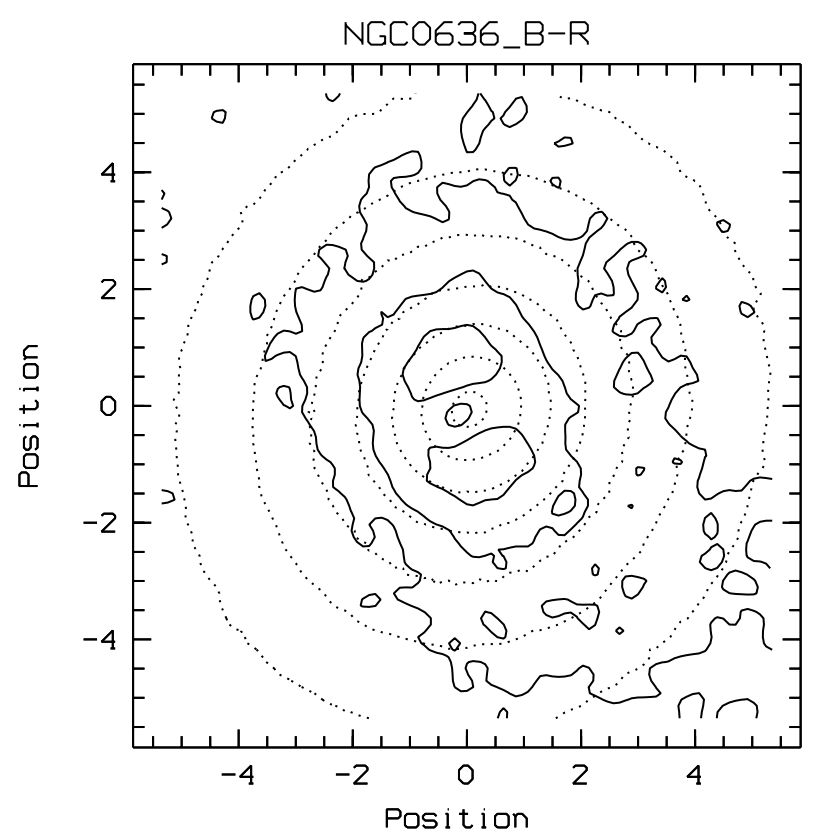

Fig. A3. NGC 0636: diEp. Positions in ". $N$ at 9 o'clock. $B-R$ isochromes: $1.525-1.575$ (0.025). $B$ isophotes: $17.0-19.0$ (0.5). The colour pattern shows a central blue feature, which seems not to be due to inadequate PSF matching

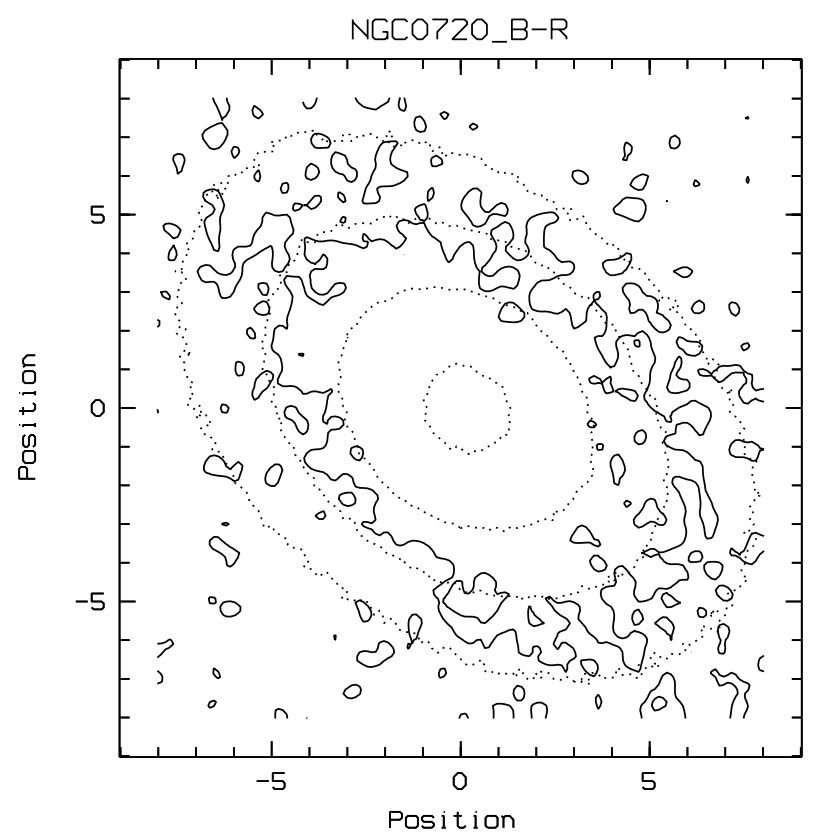

Fig. A4. NGC 0720: unE. Positions in ". $N$ at 9 o'clock. $B-R$ isochromes: $1.58-1.595(0.015)$. $B$ isophotes: $18.0-19.5$ (0.5). The colour distribution of this flat core galaxy is also remarkably flat. See also Fig. 9 


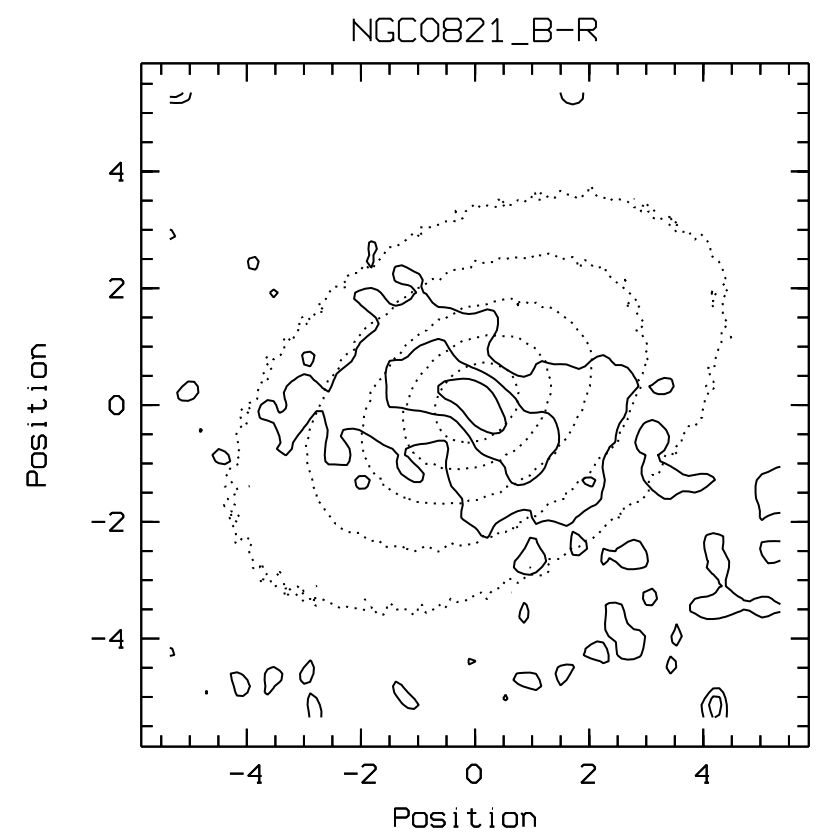

Fig. A5. NGC 0821: diE. Positions in ". $N$ at 9 o'clock. $B-R$ isochromes: $1.675-1.725$ (0.025). $B$ isophotes: $17.5-19.5$ (0.5). A low contrast red lane nearly along the minor axis is the notable feature of the modest dust pattern

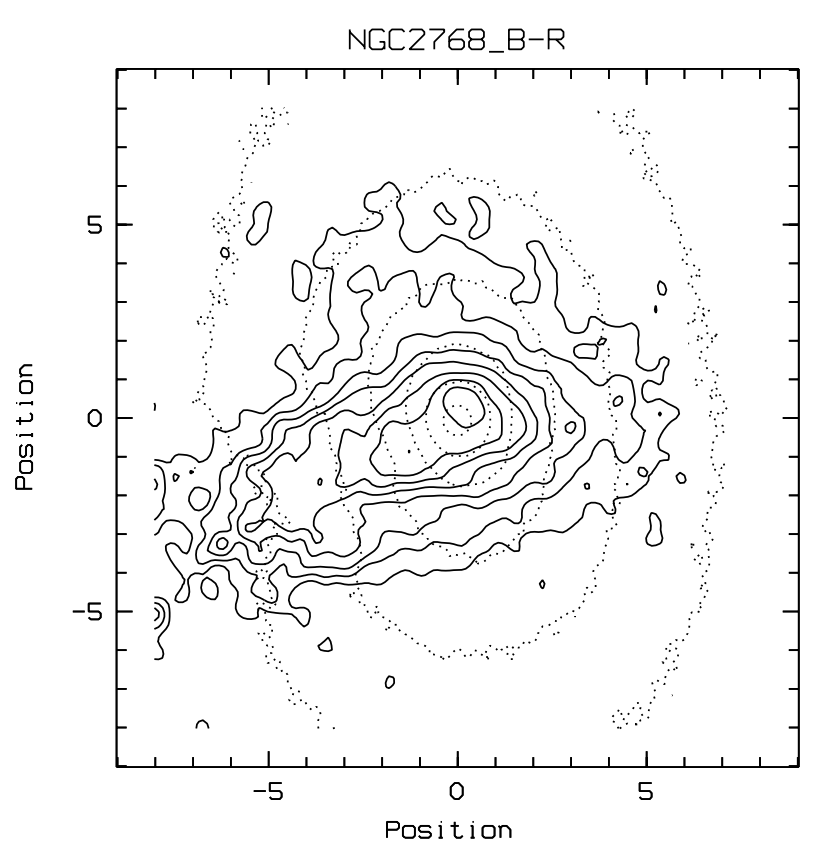

Fig. A7. NGC 2768: diE. Positions in ". $N$ at 9 o'clock. $B-R$ isochromes: $1.775-1.975$ (0.025). $B$ isophotes: $17.5-20.0$ (0.5). The object presents a complex dust pattern with extensive diffuse lanes mostly along the minor axis

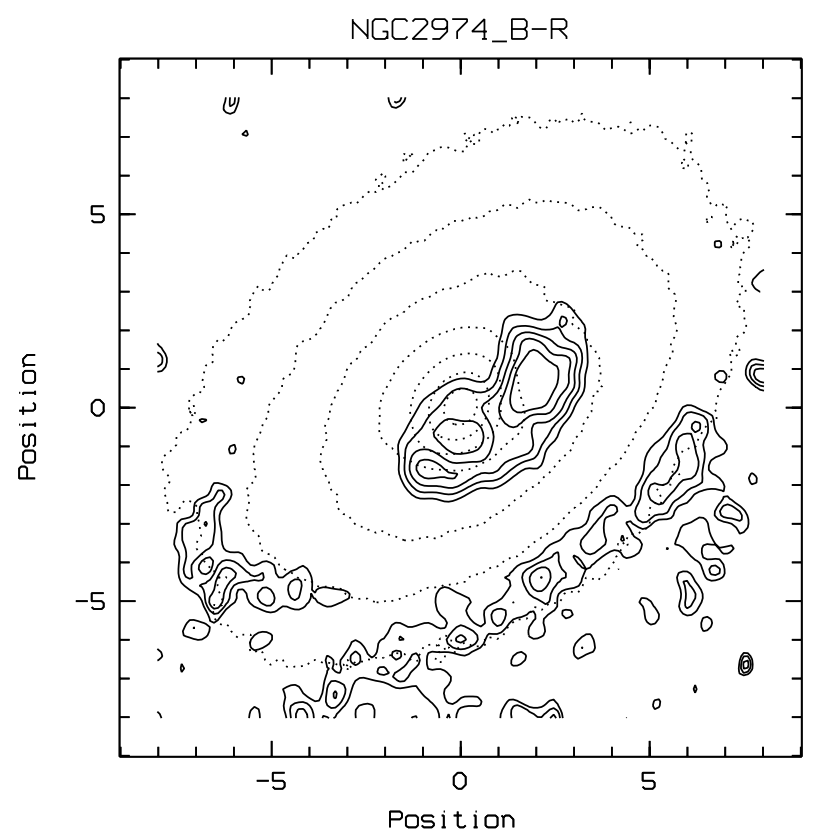

Fig. A8. NGC 2974: diE. Positions in ". $N$ at 9 o'clock. $B-R$ isochromes: $1.675-1.80(0.025)$. $B$ isophotes: $17.0-20.0$ (0.5). The object presents a complex dust pattern with multiple arclets. The peak reddening is well away from the galaxian center
Fig. A6. NGC 1052: diE. Positions in ". $N$ at 9 o'clock. $B-R$ isochromes: $1.60-1.80(0.025)$. $B$ isophotes: $17.2-19.2$ $(0.5)$. The object presents a complex dust pattern with a prominent lane near the minor axis 


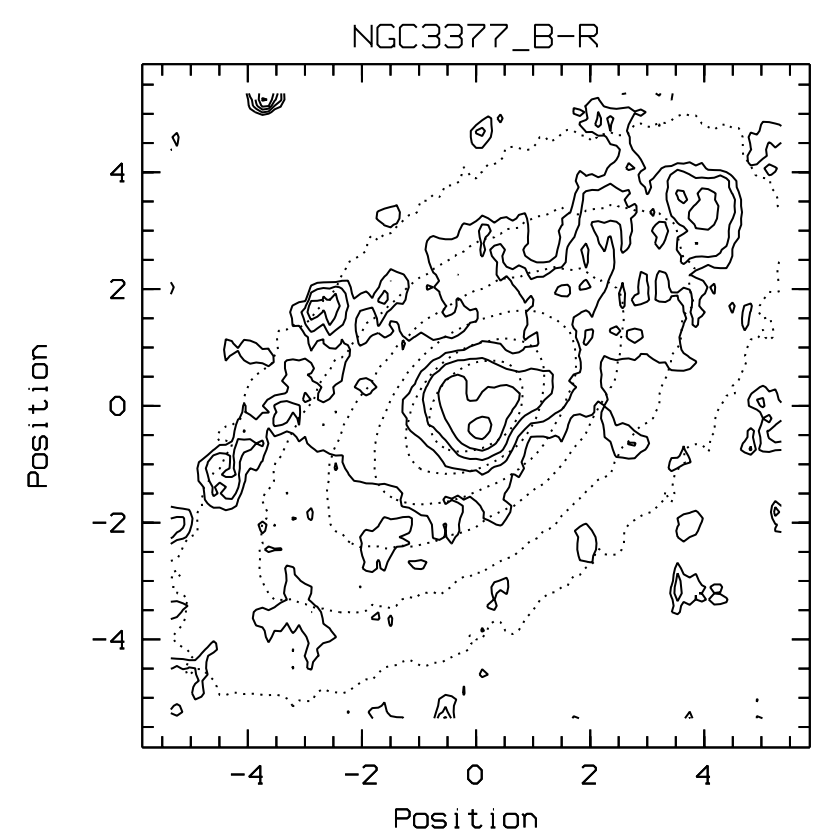

Fig. A9. NGC 3377: diE. Positions in ". $N$ at 9 o'clock. $B-R$ isochromes: $1.49-1.59$ (0.025). $B$ isophotes: $16.5-19.0$ (0.5). An asymmetric red feature is limited by a discontinuous arclet. See also HST frame in Lauer et al. (1995)

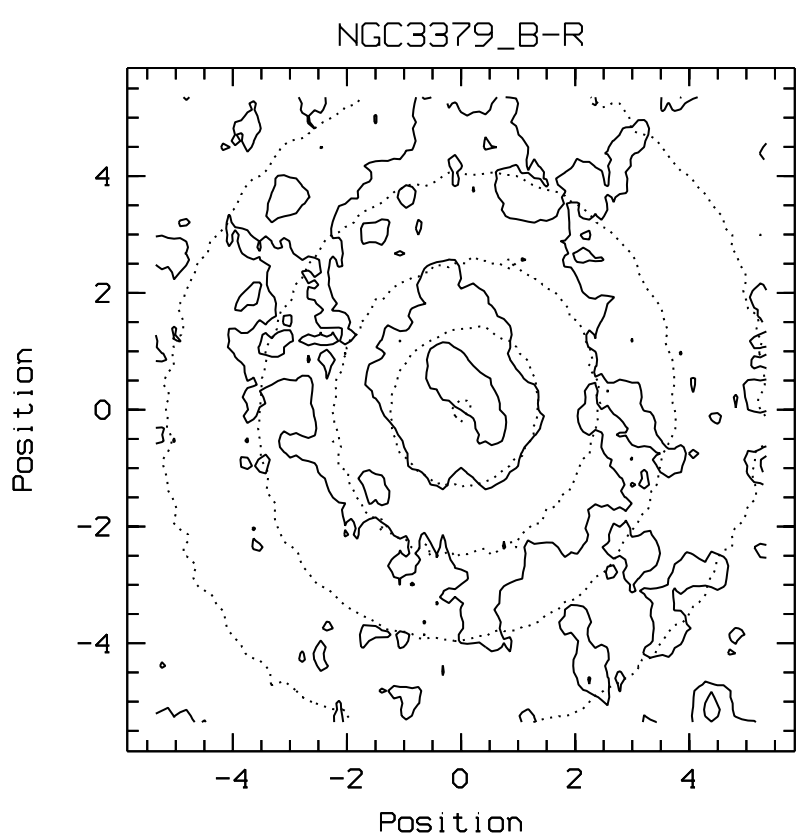

Fig. A10. NGC 3379: unE. Positions in ". $N$ at 9 o'clock. $B-R$ isochromes: $1.64-1.69(0.025)$. $B$ isophotes: $16.5-18.5$ $(0.5)$. The core red pattern is tilted with a narrow inner lane

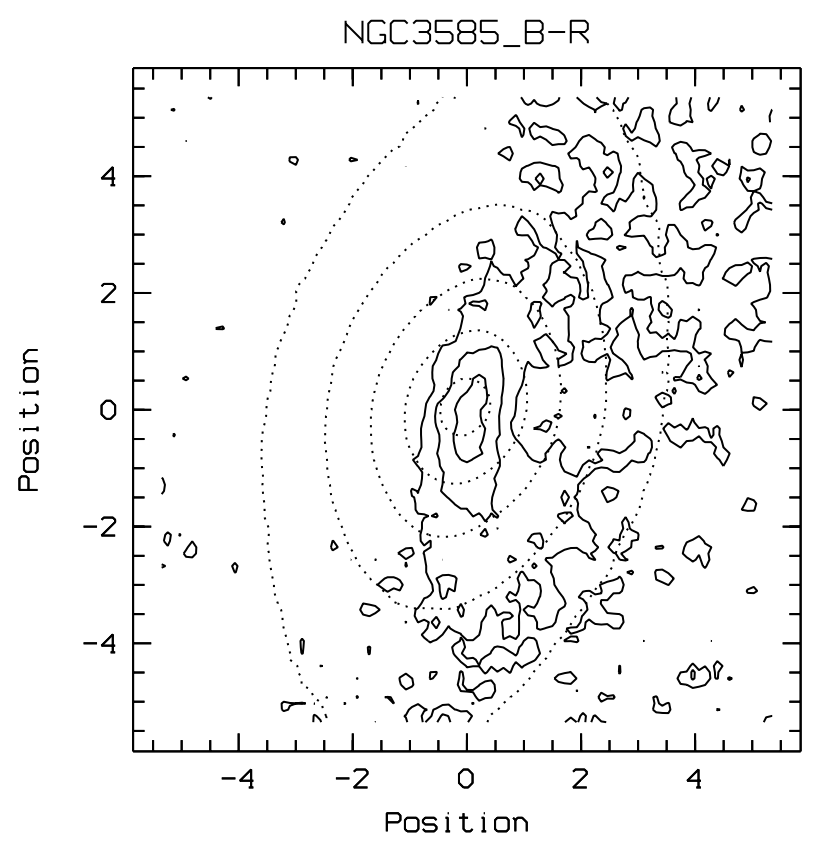

Fig. A11. NGC 3585: diE. Positions in ". $N$ at 9 o'clock. $B-R$ isochromes: $1.63-1.68$ (0.025). $B$ isophotes: $16.6-18.6$ (0.5). The flattening of the isochromes, and their shift along the minor axis, suggest some dust concentration in the inner disk

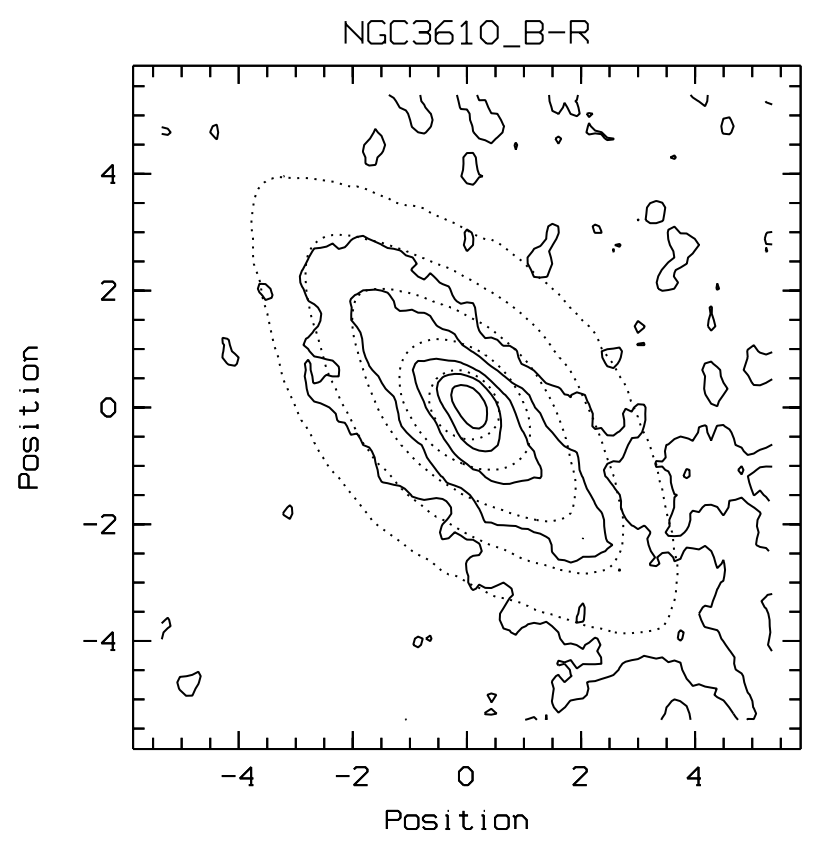

Fig. A12. NGC 3610: diE. Positions in ". $N$ at 9 o'clock. $B-R$ isochromes: $1.47-1.57$ (0.025). $B$ isophotes: $16.75-18.75$ (0.5). The flattening of the isochromes, and their shift along the minor axis, suggest some dust concentration in the inner disk 


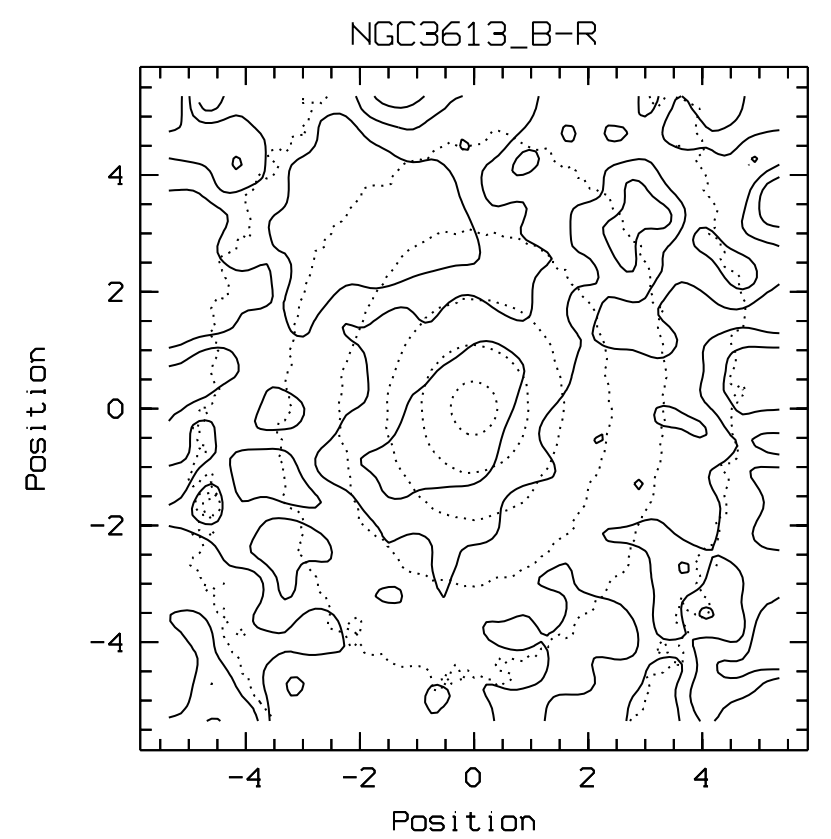

Fig. A13. NGC 3613: diE. Positions in ". $N$ at 9 o'clock. $B-R$ isochromes: $1.50-1.55$ (0.025). $B$ isophotes: $17.0-19.5$ $(0.5)$. The frames are of poor $S / N$ and small usable field. The $B-R$ pattern is of low contrast and suggests only little dust

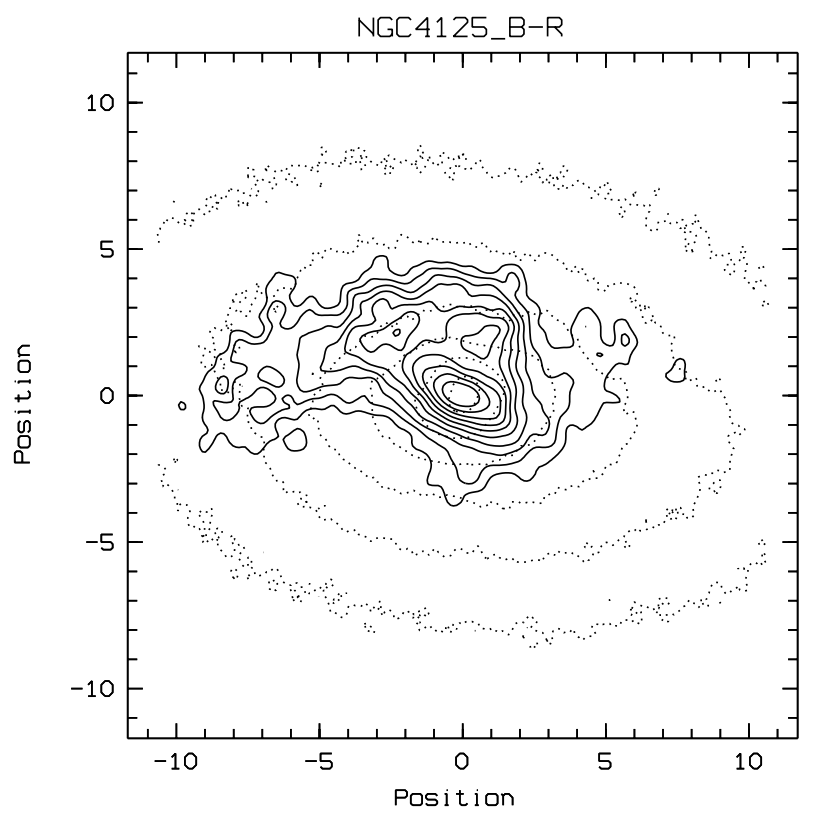

Fig. A14. NGC 4125: diE. Positions in ". $N$ at 12 o'clock. $B-R$ isochromes: $1.60-1.85(0.025)$. $B$ isophotes: $17.5-20.0$ (0.5). This object shows an important and complex dust pattern. Its colour gradient is exceptionally large also farther out than this pattern. See also Figs. 1 and 2

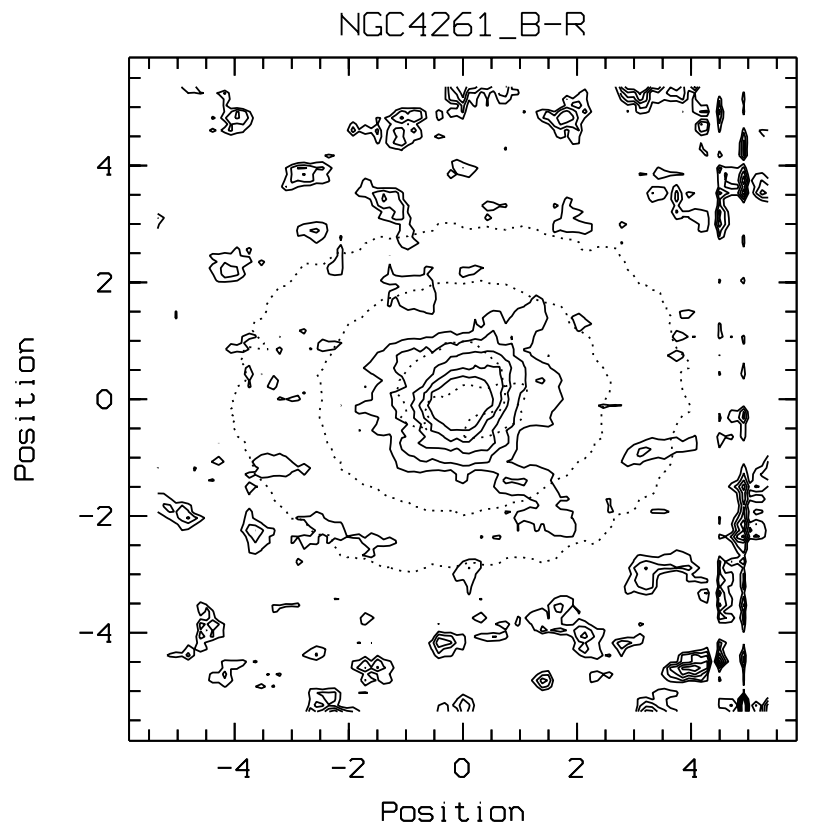

Fig. A15. NGC 4261: diE. Positions in ". $N$ at 2 o'clock. $B-R$ isochromes: $1.65-1.75$ (0.025). $B$ isophotes: $16.7-18.75$ (0.5). A small dust arclet is seen in $B$ light just below the center, and greatly disturbs the maximum $\mathrm{SuBr}$ contour here plotted

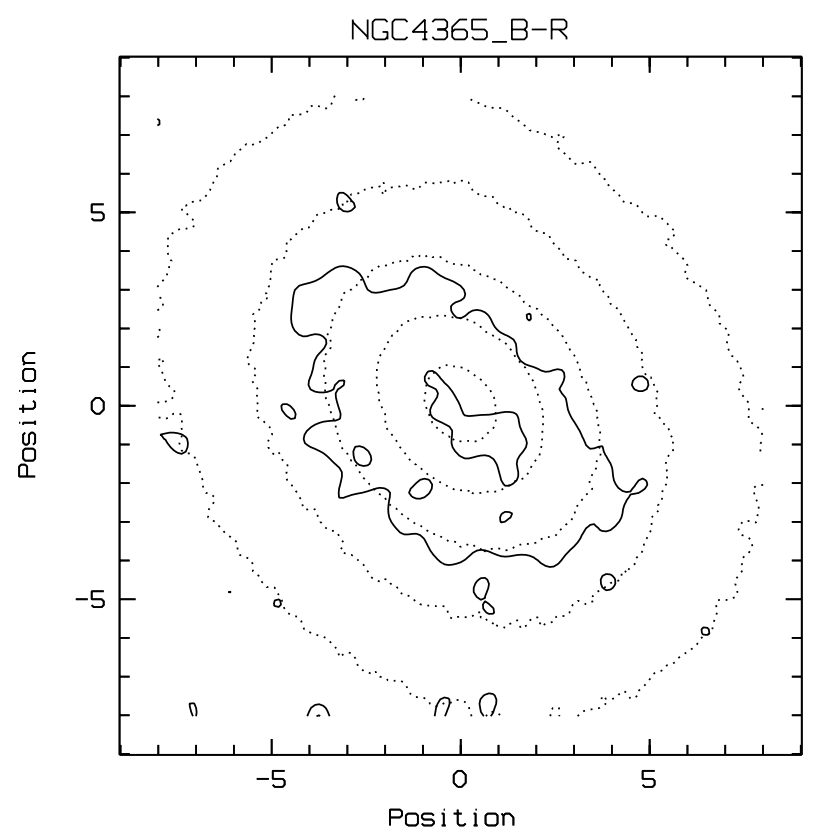

Fig. A16. NGC 4365: boE. Positions in ". $N$ at 12 o'clock. $B-R$ isochromes: $1.575-1.60(0.025) . B$ isophotes: $17.5-19.5$ (0.5). The colour distribution of this flat core galaxy is also remarkably flat. Compare with Carollo et al. (1997) 


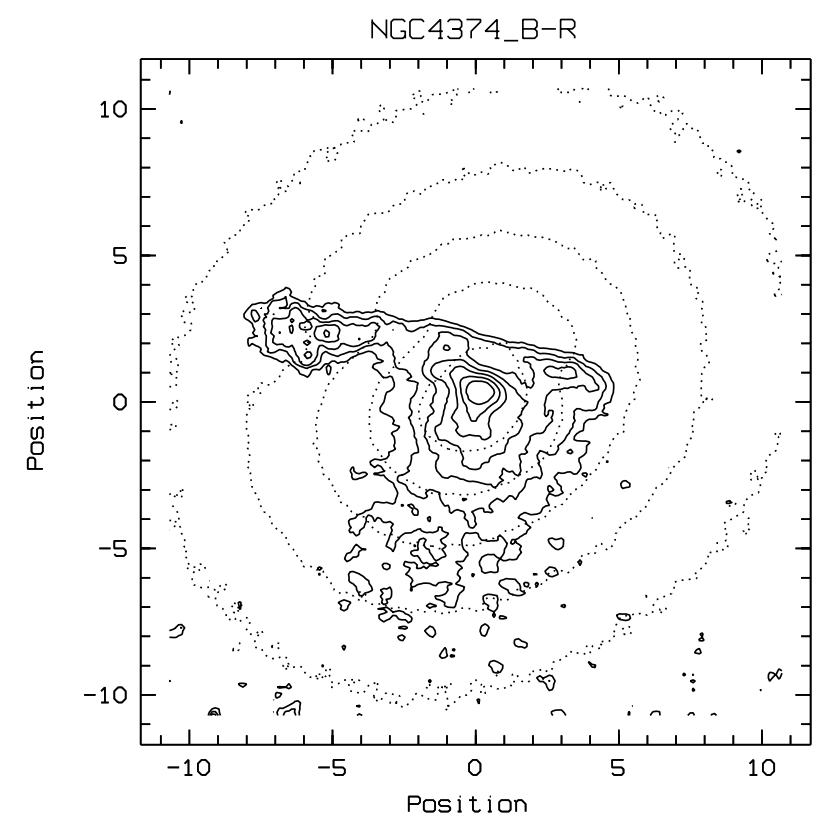

Fig. A17. NGC 4374: unE. Positions in ". $N$ at 12 o'clock. $B-R$ isochromes: $1.70-1.85(0.025)$. $B$ isophotes: $17.5-19.5$ (0.5). The large asymmetric dust pattern is sharply bounded northwards by a prominent lane

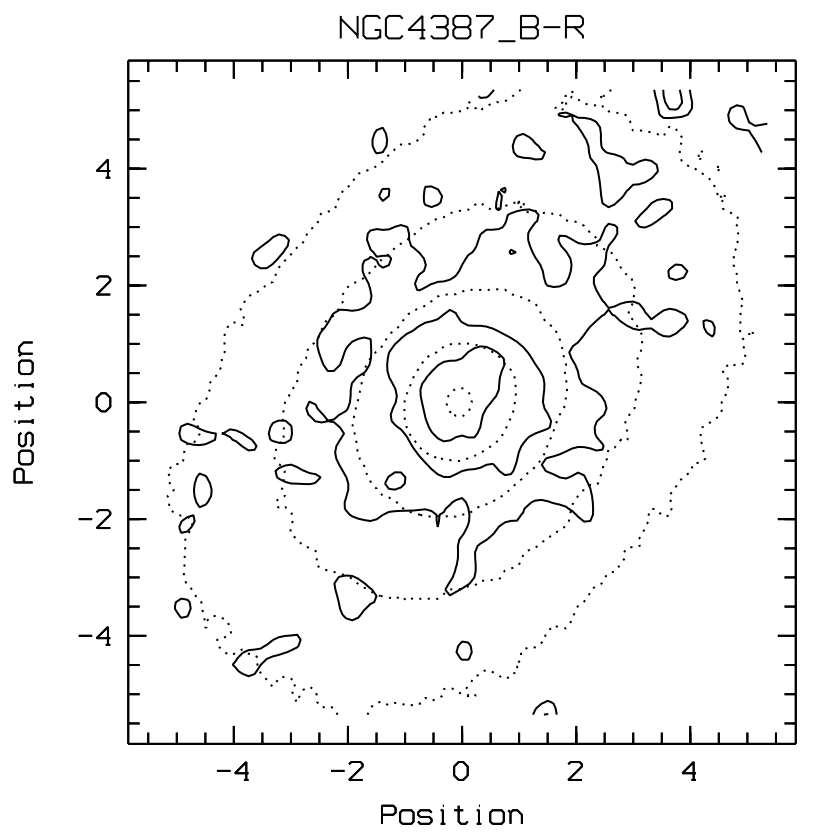

Fig. A18. NGC 4387: boE. Positions in ". $N$ at 12 o'clock. $B-R$ isochromes: $1.585-1.66$ (0.025). $B$ isophotes: $18.2-19.7$ (0.5). This boE, a member of the family of rotationally supported minor objects, has no significant colour pattern

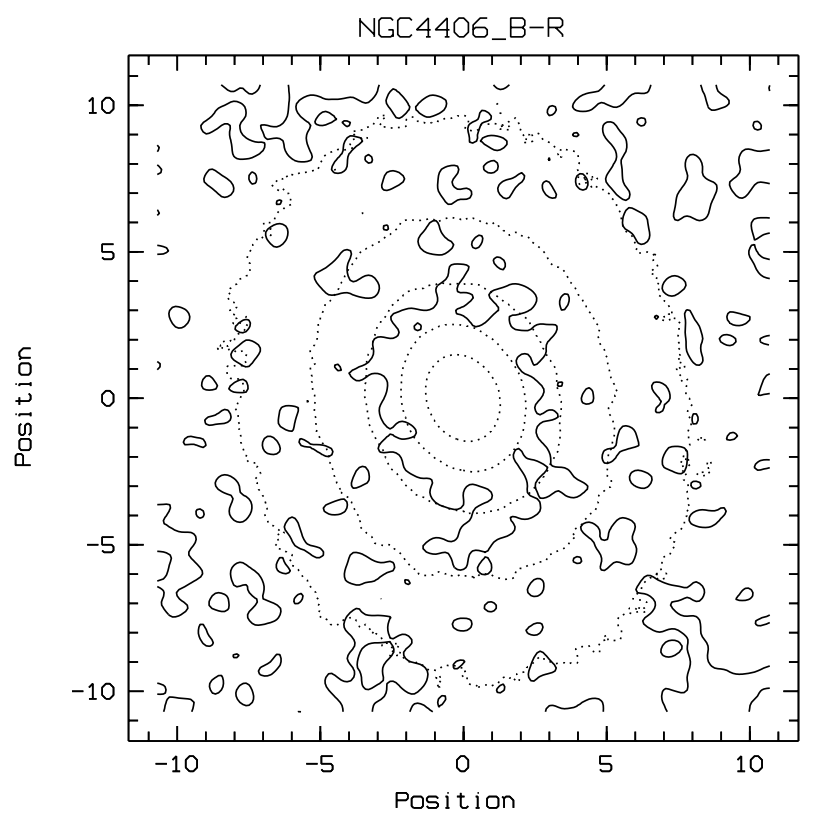

Fig. A19. NGC 4406: boE. Positions in ". $N$ at 12 o'clock. $B-R$ isochromes: $1.58-1.595$ (0.015). $B$ isophotes: $17.0-19.5$ (0.5). The colour distribution of this flat core galaxy is also remarkably flat. See also Fig. 9, and the $V-I$ data by Carollo et al. (1997)

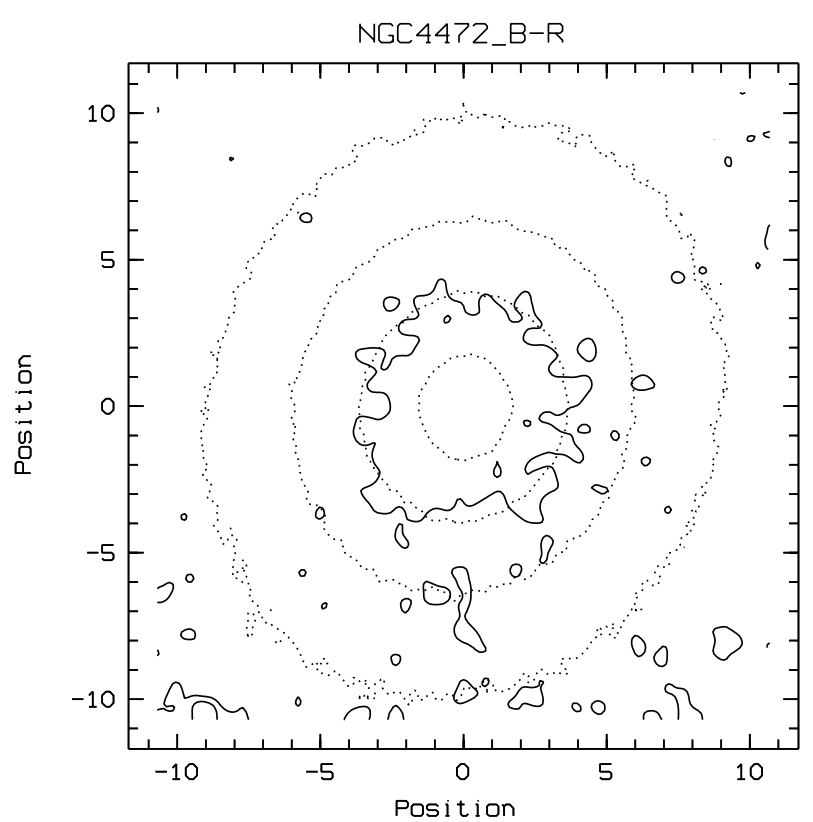

Fig. A20. NGC 4472: unE. Positions in ". $N$ at 12 o'clock. $B-R$ isochromes: $1.62-1.63(0.01)$. $B$ isophotes: $17.0-18.5$ (0.5). The colour distribution of this flat core galaxy is also remarkably flat. See also Fig. 9 


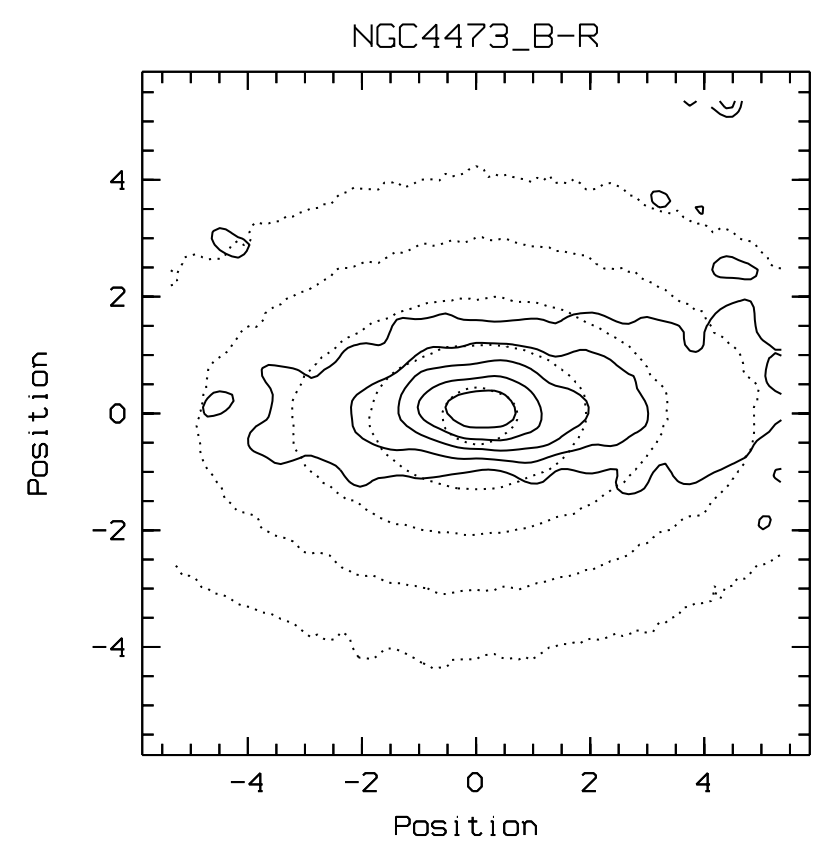

Fig. A21. NGC 4473: diE. Positions in ". $N$ at 12 o'clock. $B-R$ isochromes: $1.575-1.675$ (0.025). $B$ isophotes: $16.75-$ 18.75 (0.5). The flattening of the isochromes, and their shift along the minor axis, suggest dust concentration in the disk

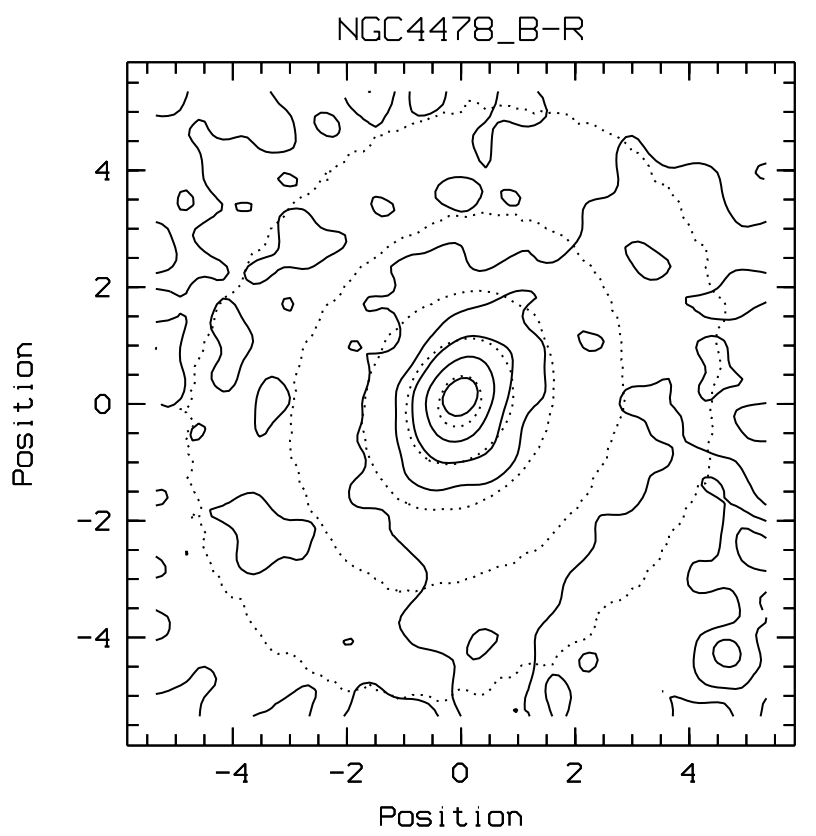

Fig. A22. NGC 4478: boE. Positions in ". $N$ at 12 o'clock. $B-R$ isochromes: $1.575-1.675$ (0.025). $B$ isophotes: $17.25-$ $18.25(0.5)$. This boE, a member of the family of rotationally supported minor objects, has no significant colour pattern, besides a rather sharp central red peak

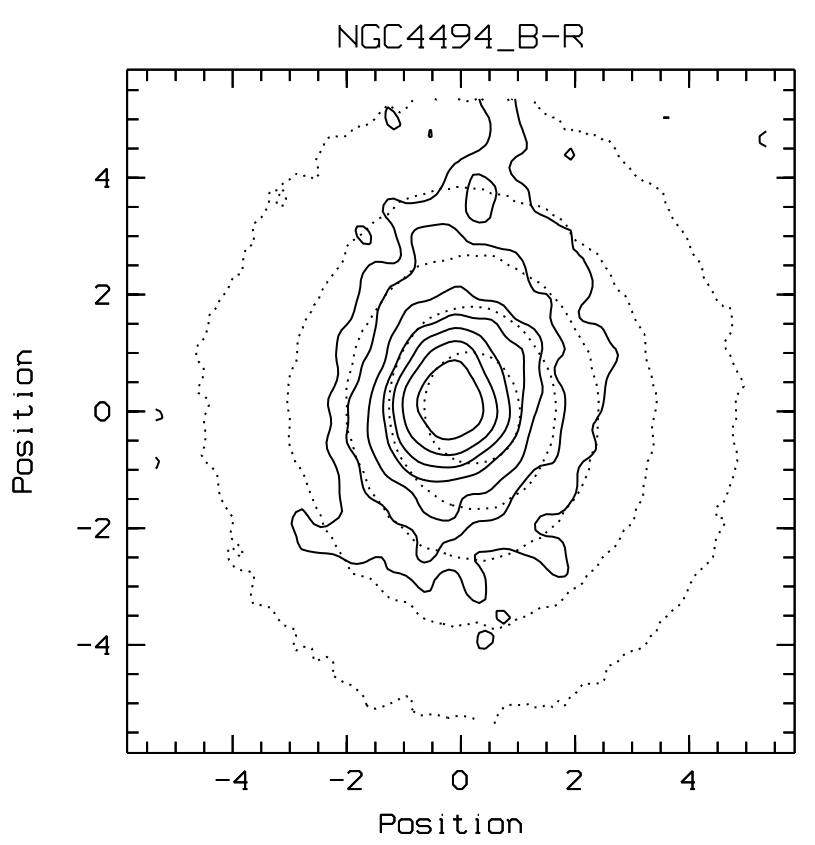

Fig. A23. NGC 4494: unE. Positions in ". $N$ at 12 o'clock. $B-R$ isochromes: $1.45-1.60$ (0.025). $B$ isophotes: $17.0-19.0$ (0.5). This slightly disky object shows no obvious colour pattern, but for a sharp nearly cemtral red peak. HST V-I images disclose a small dust ring (see text)

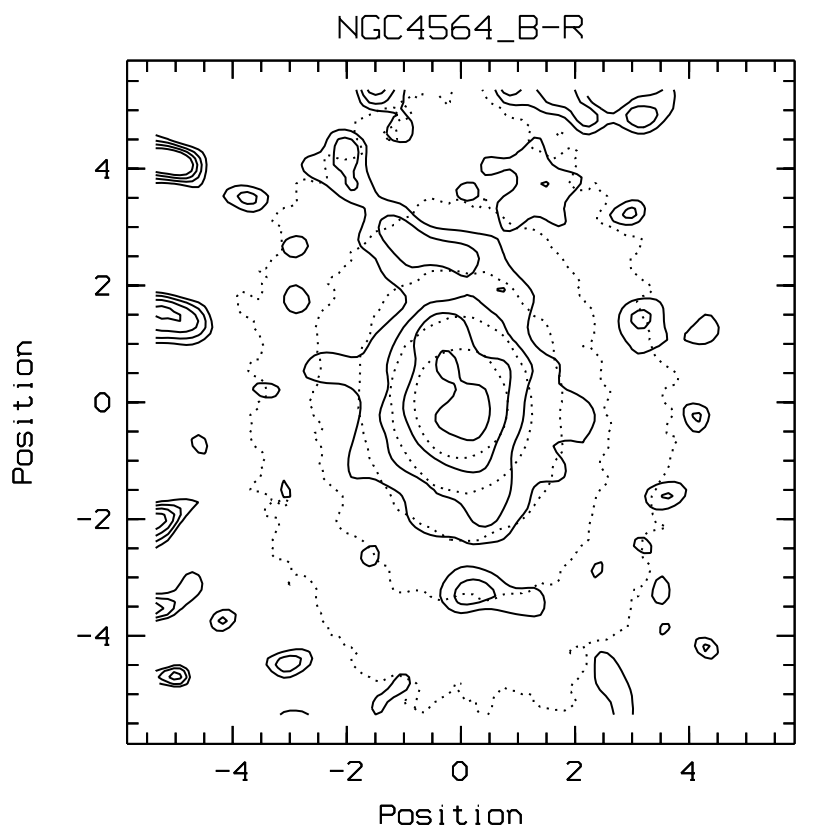

Fig. A24. NGC 4564: diE. Positions in ". $N$ near 2 o'clock $B-R$ isochromes: $1.60-1.70(0.025)$. $B$ isophotes: $17.2-19.2(0.5)$. Poor $S / N$. Possible faint, near central, colour pattern. The disk is clearly seen farther than $10^{\prime \prime}$ along the major axis, with possible dust concentration (see Paper II) 


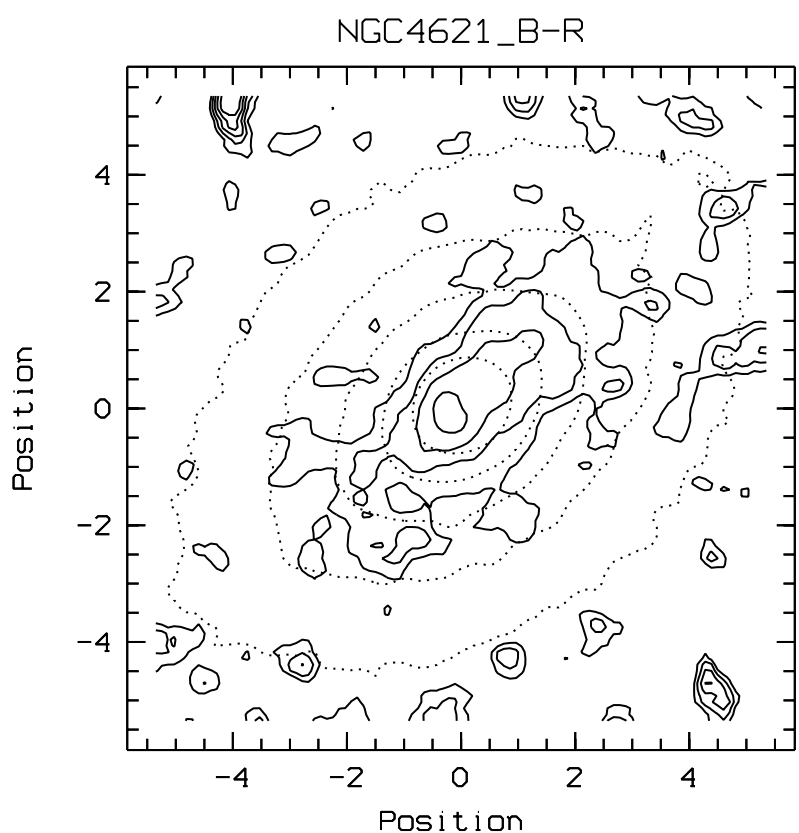

Fig. A25. NGC 4621: diE. Positions in ". $N$ near 8 o'clock. $B-R$ isochromes: $1.595-1.695$ (0.025). $B$ isophotes: $16.75-$ 18.25 (0.5). Poor $S / N$. The flattening of the inner isochromes suggests some dust concentration in the disk

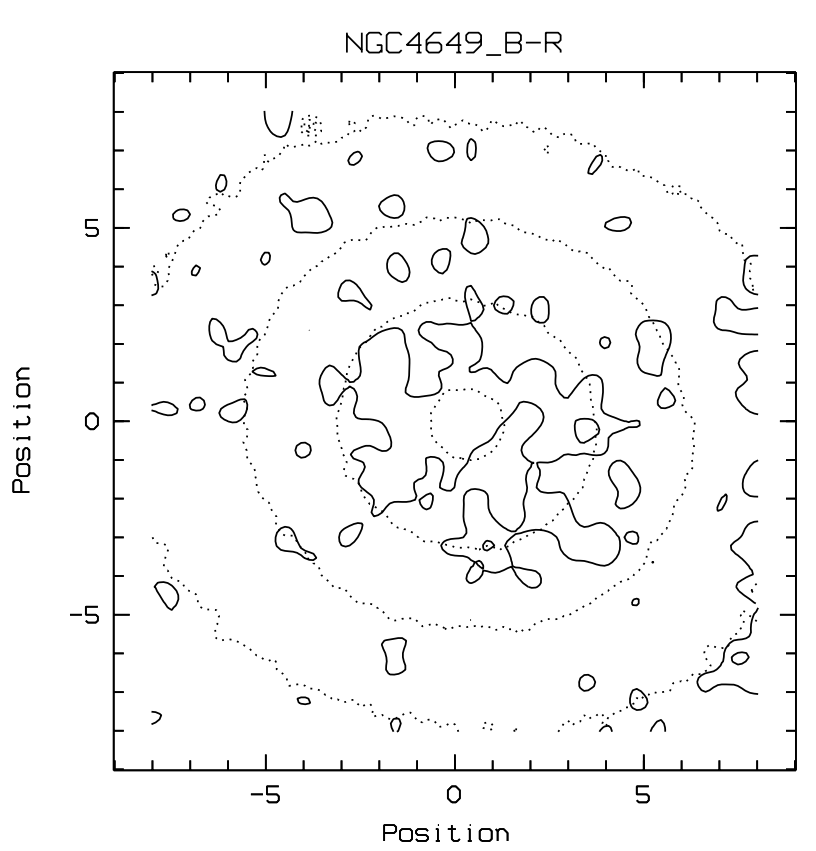

Fig. A26. NGC 4649: boE. Positions in ". $N$ near 1 o'clock. $B-R$ isochromes: $1.655-1.665$ (0.01). $B$ isophotes: $17.0-19.0$ (0.5). Poor $S / N$. The colour distribution of this flat core galaxy is also remarkably flat. See Fig. 9

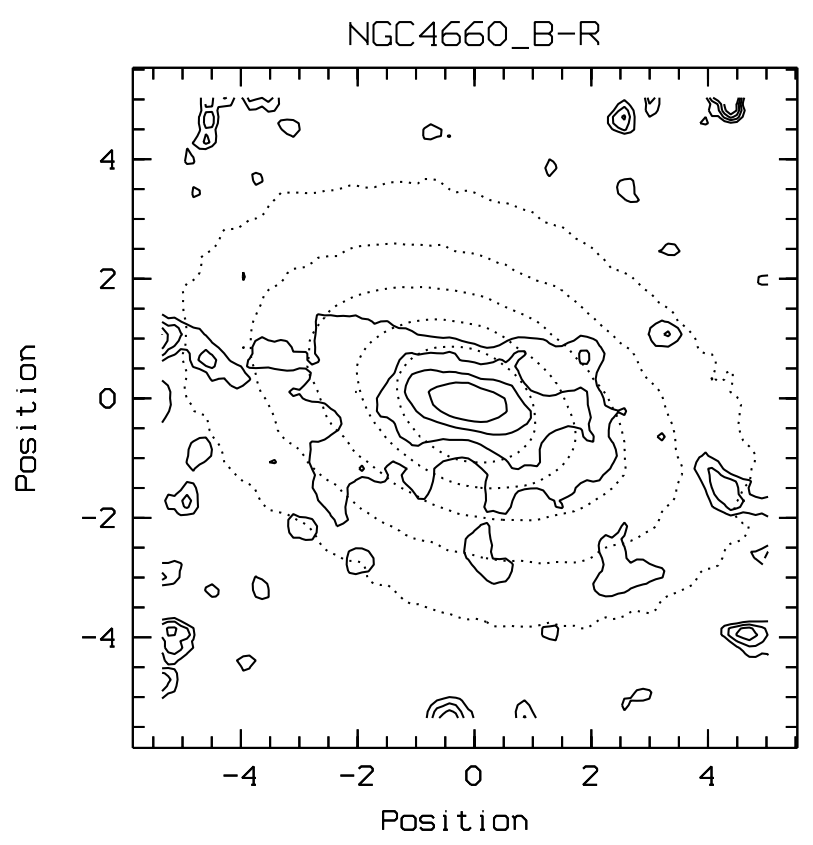

Fig. A27. NGC 4660: diE. Positions in ". $N$ near 1 o'clock. $B-R$ isochromes: $1.55-1.65$ (0.025). $B$ isophotes: $17.0-19.0$ (0.5). The flattening of the inner isochromes suggesgts some dust concentration in the disk, while their tilt might indicate a faint dust lane crossing the core

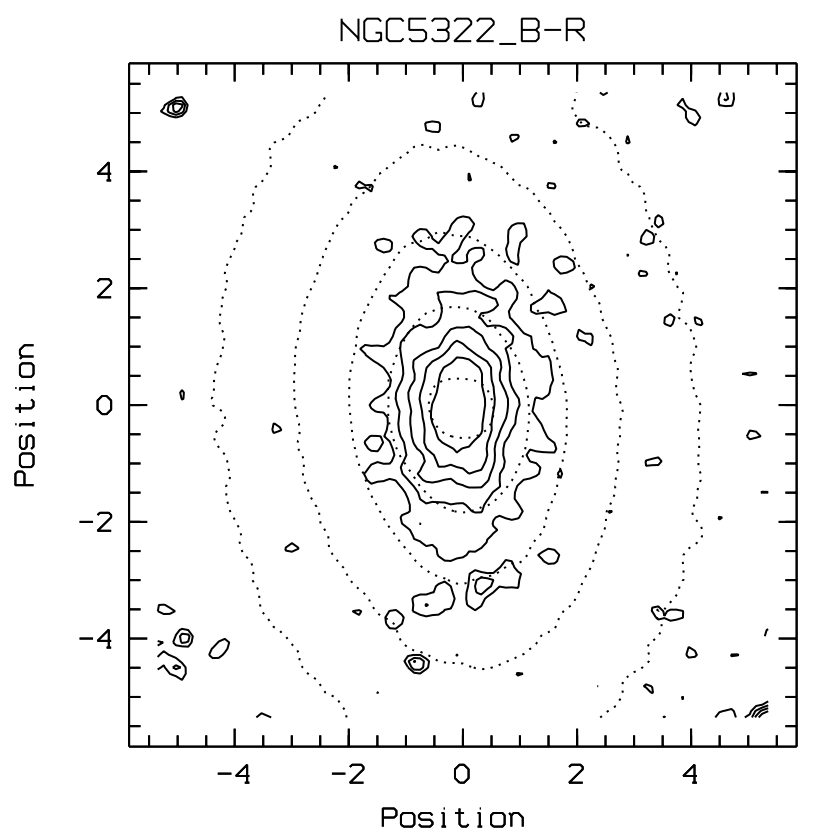

Fig. A28. NGC 5322: boE. Positions in ". $N$ near 3 o'clock. $B-R$ isochromes: $1.45-1.55$ (0.025). $B$ isophotes: $17.0-19.0$ (0.5). The flattening of the inner isochromes suggests some dust concentration in the inner "decoupled" disk. See also the HST frame in Lauer et al. (1995) 


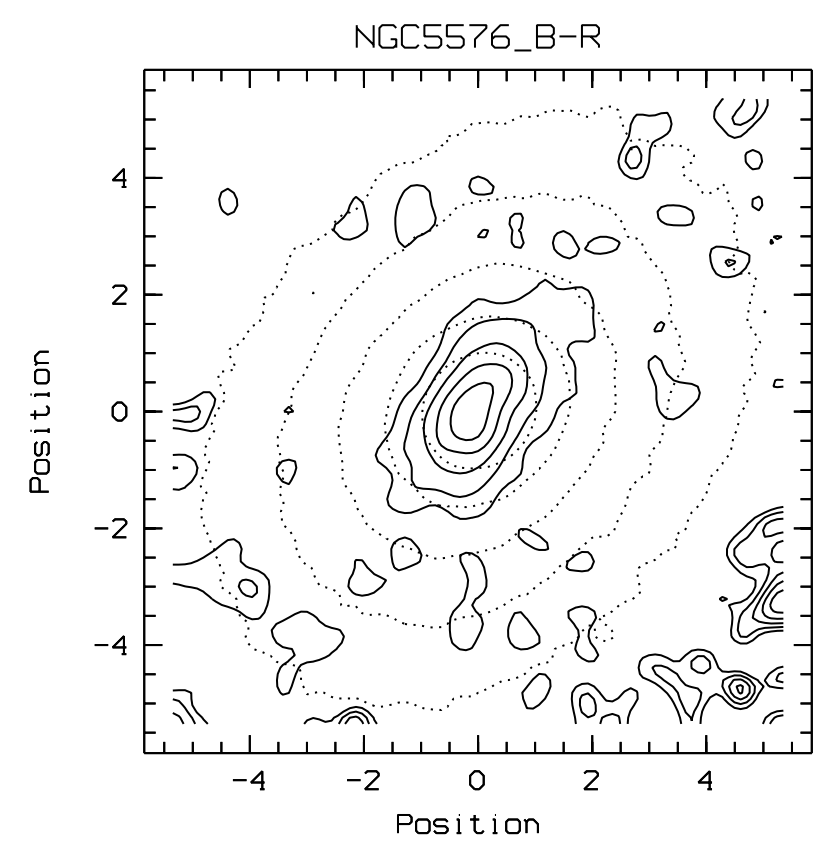

Fig. A29. NGC 5576: boEp. Positions in ". $N$ near 10 o'clock. $B-R$ isochromes: $1.425-1.55$ (0.025). $B$ isophotes: $17.0-19.0$ (0.5). Poor $S / N$. No colour pattern is seen, but for the rather sharp central peak

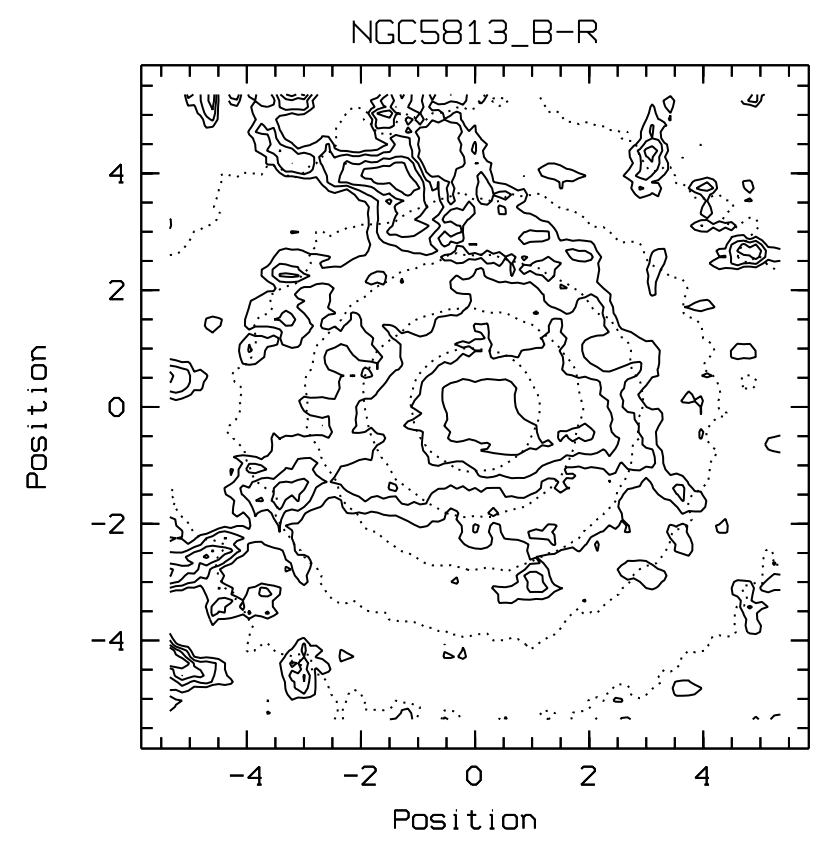

Fig. A30. NGC 5813: unE. Positions in ". $N$ near 2 o'clock. $B-R$ isochromes: $1.60-1.70$ (0.025). $B$ isophotes: $18.0-20.0$ $(0.5)$. Poor $S / N$. An asymmetric core dust pattern is present

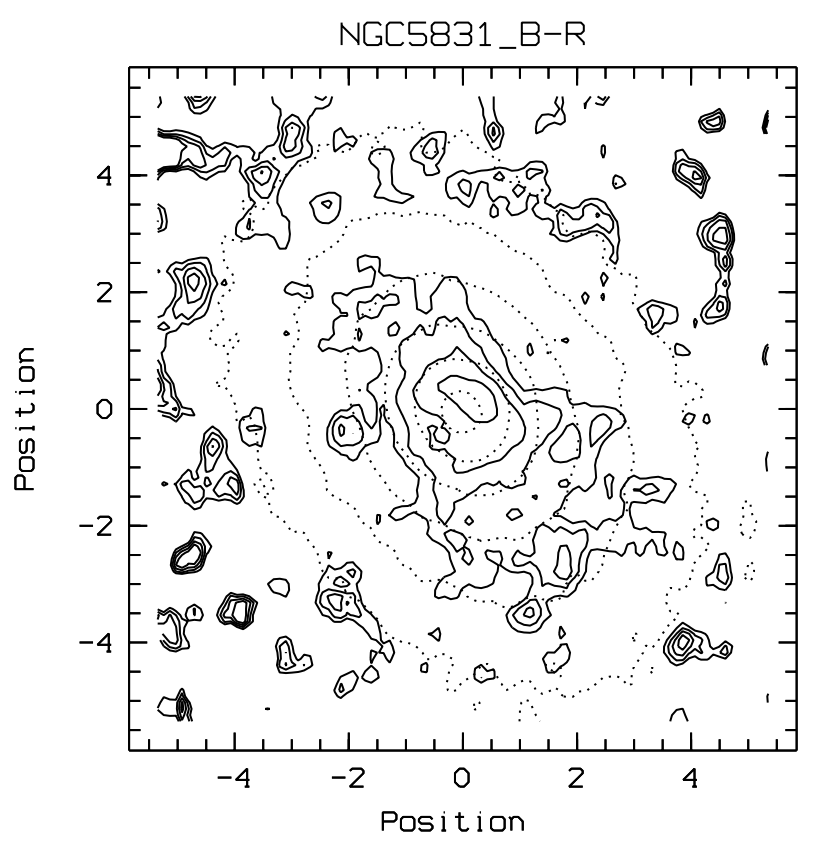

Fig. A31. NGC 5831: diE. Positions in ". $N$ near 3 o'clock. $B-R$ isochromes: $1.61-1.71$ (0.025). $B$ isophotes: $17.4-19.4$ (0.5). Poor $S / N$. An asymmetric core dust pattern is present, again with some indication of flattening of the isochromes

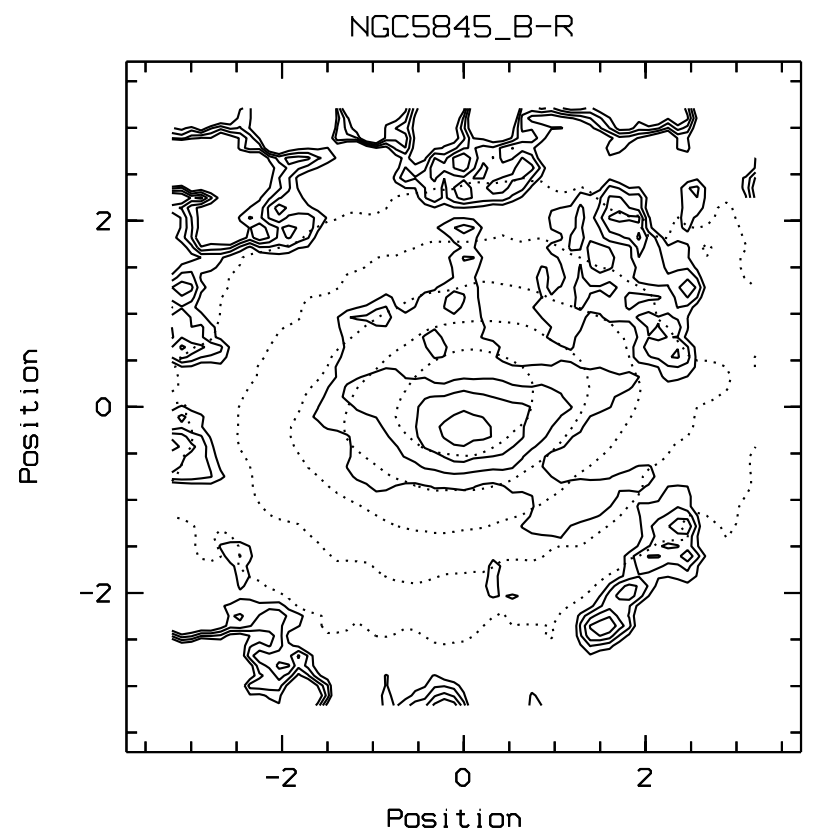

Fig. A32. NGC 5845: diE. Positions in ". $N$ near 3 o'clock. $B-R$ isochromes: $1.60-1.70(0.025)$. $B$ isophotes: $17.0-19.0$ (0.5). Poor $S / N$. The flattening of the inner isochromes suggesgts some dust concentration in the inner "decoupled" disk. See also the HST frame in Lauer et al. (1995) 